\title{
MODULES AND STABILITY THEORY
}

\author{
ANAND PILLAY AND MIKE PREST
}

\begin{abstract}
Modules are now widely recognized as important examples of stable structures. In fact, in the light of results and conjectures of Zilber [Zi] ( $\aleph_{1}$-categorical structures are "field-like", "module-like" or "trivial"), we may consider modules as one of the typical examples of stable structures. Our aim here is both to prove some new results in the model theory of modules and to highlight the particularly clear form of, and the algebraic content of, the concepts of stability theory when applied to modules.

One of the main themes of this paper is the connection between stabilitytheoretic notions, such as ranks, and algebraic decomposition of models.

We will usually work with $T$, a complete theory of $R$-modules, for some ring $R$. In $\S 2$ we show that the various stability-theoretic ranks, when defined, are the same. In $\S 3$ we show that $T$ (not necessarily superstable) is nonmultidimensional (in the sence of Shelah [Sh1]). In $\S 4$ we consider the algebraic content of saturation and we show, for example, that if $M$ is a superstable module then $M$ is $F_{\aleph_{0}}^{a}$-saturated just if $M$ is pure-injective and realizes all types in finitely many free variables over $\phi$. In $\S 5$ we use our methods to reprove Ziegler's theorem on the possible spectrum functions. In $\S 6$ we show the profusion (in a variety of senses) of regular types. In $\S 7$ we give a structure theorem for the models of $T$ in the case where $T$ has $U$-rank 1 .
\end{abstract}

It is unavoidable that we must assume some familiarity with certain fundamental ideas of the subject. The main points are described briefly in $\S 0$, but for more detail, the reader my consult $[\mathbf{P P}, \mathbf{P t 1}, \mathbf{P t 2}, \mathbf{Z g}]$.

0. Preliminaries and prerequisites. Throughout the paper, "module" means right $R$-module, where $R$ is a fixed ring with 1 ; the language is that appropriate for $(R-)$ modules; and $T$ always will denote a complete theory of modules. Recall that the language has: "+" for addition in modules; " 0 " for the zero element of a module; " $-\times r$ " for multiplication by $r(r \in R)$. A positive primitive, or $\mathrm{pp}$, formula is one built up from the atomic formulas (i.e. $R$-linear equations) using $\wedge$ and $\exists$. Such formulas express solvability of matrix equations. The key result of the subject says that arbitrary formulas cannot be much more complex than pp formulas.

THEOREM 0.1 (BAUR [Ba]). Let $T$ be a complete theory of modules, and let $\chi(\bar{x})$ be any formula. Then there is $\psi(\bar{x})$, a boolean combination of $p p$ formulas, such that $T \vdash \chi(\bar{x}) \leftrightarrow \psi(\bar{x})$.

This implies that the map which takes a type $p \in S(A)$ to its $p p$-part, $p^{+}=$ $\{\varphi \in p: \varphi$ is pp $\}$ is $1-1$ - for we can recover $p$ from $p^{+}: p^{+} \cup \neg p^{-}(=\{\neg \varphi: \varphi$ is pp in $\left.\left.\mathfrak{L}_{A}, \varphi(x) \notin p(\bar{x})\right\}\right) \vdash p$ (modulo $T$ ). The set $p^{+}$is a $p p$-type. Here $S(A)$ denotes

Received by the editors May 31, 1984.

1980 Mathematics Subject Classification (1985 Revision). Primary 03C45; Secondary 16A45. 
the set of $n$-types over $A$, for some $n \geq 1 ; S_{n}(A)$ is used for definiteness (and $T$ is understood). $\mathfrak{L}_{A}$ of course is the basic language extended with constants for the elements of $A$.

Note that if $p \in S(0)$, then the pp-type $p^{+}$does not contain any but the most trivial sentences of $T$, and it is quite possible to find types over other complete theories with the same pp-part. It should not be surprising then, that certain aspects of the type $p$ are independent of the over-theory $T$.

In particular, if $p^{+}$is a pp-type over 0 perhaps in infinitely many variables, then there is associated to $p^{+}$its hull $N\left(p^{+}\right)$or just $N(p)$. This is a pure-injective $(=$ algebraically compact) module: that is, every pp-type over $N(p)$ which is finitely satisfied in $N(p)$, is actually realized in $N(p)$. Moreover, $N(p)$ is small over a realization of $p^{+}$: that is, there is $\bar{a}$ in $N(p)$ with $\operatorname{tp}^{N(p)}(\bar{a})^{+}=p^{+}$, such that, if $\bar{b}$ is in $N(p)$, then $\operatorname{tp}^{N(p)}(\bar{b} / \bar{a})^{+}$is maximal in $\left\{\operatorname{tp}^{N_{1}}(\bar{c} / \bar{a})^{+}: \bar{c}\right.$ in $N_{1}^{+} \succ N(p)$, $l(\bar{c})=l(\bar{b})\}$ : in particular, if $\bar{b} \neq \overline{0}$, then there is a pp formula $\varphi$ with $N(p) \vDash$ $\varphi(\bar{b}, \bar{a}) \wedge \neg \varphi(\overline{0}, \bar{a})(\wedge \neg \varphi(\bar{b}, \overline{0}))$. Here $\operatorname{tp}^{M}(\bar{b} / A)=\{\chi(\bar{x}, \bar{a}): \bar{a}$ in $A$ and $M \vDash \chi(\bar{b}, \bar{a})\}$, and we omit $A$ if $A=0$. Also we write $M \prec_{1}^{+} N$ if, for all $\bar{a}$ in $M, \operatorname{tp}^{M}(\bar{a})^{+}=$ $\operatorname{tp}^{N}(\bar{a})^{+}$. This hull is unique in that, if $q \in S^{T^{\prime}}(0)$ with $q^{+}=p^{+}$, if $\bar{c}$ realizes $q$ in a pure-injective model $N$ of $T^{\prime}$, then there is a pure embedding $f: N(p)=N(\bar{a}) \hookrightarrow N$ which, since $N(p)$ is pure-injective, is actually split, with $f \bar{a}=\bar{c}$ : moreover, the image of $f$ is a minimal direct summand of $N$ containing $\bar{c}$.

In the particular case where $A \prec_{1}^{+} \tilde{M}$, where $\tilde{M}$ denotes the monster model, $N(A)$ is denoted $\bar{A}$, and termed the pure-injective hull of $A$, and one has $A \prec \bar{A}$ [Sg1]. Note that if $M \vDash T$ is $|T|^{+}$-saturated, then $M$ is pure-injective (this follows easily from the comments following).

If $\varphi(\bar{x})$ is a pp formula over 0 , and $M$ is a module, then $\varphi(M)=\left\{\bar{a} \in M^{l(\bar{x})}\right.$ : $M \vDash \varphi(\bar{a})\}$ is a subgroup of $M^{l(\bar{x})}$ (where $l(\bar{x})$ is the length of the tuple $\bar{x}$ ): this is a direct consequence of linearity of pp formulas. If $\varphi$ is a pp formula with parameters, say $\varphi$ is $\varphi(\bar{x}, \bar{a})$, then $\varphi(M, \bar{a})$ is a coset of the pp-definable subgroup $\varphi(M, \overline{0})$. If $\varphi(\bar{x}), \psi(\bar{x})$ are pp formulas then $\varphi(M) \cap \psi(M)$, respectively $\varphi(M)+\psi(M)$, are defined by the pp formulas $\varphi \wedge \psi$, resp., $\varphi+\psi$, where $(\varphi+\psi)(\bar{x})$ is $\exists \bar{x}_{1}, \bar{x}_{2}\left(\varphi\left(\bar{x}_{1}\right) \wedge\right.$ $\left.\psi\left(\bar{x}_{2}\right) \wedge \bar{x}=\bar{x}_{1}+\bar{x}_{2}\right)$. We will also have occasion to consider subgroups (of the monster model $\tilde{M}$ ) defined by possibly infinite sets of pp formulas.

The next result is used frequently in showing that certain sets of formulas are consistent. The result is due to B. H. Neumann (see e.g. $[\mathbf{R}]$; for the bounds see [Mo, Ho]).

Neumann's Lemma 0.2. Suppose $H, H_{1}, \ldots, H_{n}$ are all subgroups of a group. Suppose $a+H \subseteq \bigcup_{i=1}^{n} a_{i}+H_{i}$. Then

$$
a+H \subseteq \bigcup\left\{a_{i}+H_{i}:\left[H: H \cap H_{i}\right] \leq n !\right\}
$$

The Baur-Garavaglia-Monk invariants are the $\operatorname{Inv}(T, \varphi, \psi)=\operatorname{Inv}(M, \varphi, \psi)=$ $[\varphi(M): \varphi(M) \cap \psi(M)]$ (finite or " $\infty$ "), where $\varphi, \psi$ are pp formulas and $M$ is a model of $T$ : note that these do not depend on choice of model. The next result is used frequently, particularly in conjunction with observations such as $\operatorname{Inv}(M \oplus N, \varphi, \psi)=$ $\operatorname{Inv}(M, \varphi, \psi) \cdot \operatorname{Inv}(N, \varphi, \psi)$. 
THEOREM 0.3 (CF. [Ba]). If $M, N$ are modules then $M \equiv N$ iff $\operatorname{Inv}(M, \varphi, \psi)$ $=\operatorname{Inv}(N, \varphi, \psi)$, for all pp formulas (in one free variable).

The following also is used without explicit mention.

THEOREM 0.4 [Sg1]. If $M, N$ are modules with $M \prec_{1}^{+} N$ and $M \equiv N$, then $M \prec N$.

Notationally, we will identify pp formulas with the subgroups and cosets they define. Thus $[\varphi: \psi]$ is the index of subgroups $\psi(M)$ in $\varphi(M)$ where $M$, if not clear from context, may as well be taken to be $\tilde{M}$.

Given a type $p$, we may think of $p^{+}$as a filter of cosets (of definable subgroups of the monster model $\tilde{M}$ ). The corresponding filter of subgroups, or better, the closure of this under definable subgroups of finite index, is an important invariant of $p$, measuring the extent to which $p$ is "restricted" (see $[\mathbf{P o}, \mathbf{P t 2}, \mathbf{Z g}]$ ).

Definition 0.5. Suppose $p \in S(A)$. Set $\mathcal{G}(p)=\left\{\varphi(\bar{x}, \overline{0}): \varphi(\bar{x}, \bar{a}) \in p^{+}\right.$for some $\bar{a}$ in $A$ \}. Identifying formulas with the sets they define, we may regard $\mathcal{G}(p)$ as a filter of subgroups (of $\tilde{M}^{l(\vec{x})}$ ). Therefore set $G(p)=\bigcap \mathcal{G}(p)$-a possibly infinite definable subgroup of $\tilde{M}^{l(\bar{x})}$. Also let $\mathcal{G}_{0}(p)=\{\psi(\bar{x}):[\varphi: \varphi \wedge \psi]$ is finite for some $\varphi \in \mathcal{G}(p)\}$ and set $G_{0}(p)=\bigcap \mathcal{G}_{0}(p)$. The next lemma says that $G_{0}(p)$ is "connected"--that is, has no proper definable subgroups of finite index. The proof is an easy compactness argument. Note that from 0.2 it is clear that a connected definable subgroup is pp-definable.

LEMMA 0.6. Let $p \in S(A)$ and suppose $H$ is a pp-definable subgroup. If $\left[G_{0}(p): G_{0}(p) \cap H\right]$ is finite, then $H \in \mathcal{G}_{0}(p)$ (so $H \geq G_{0}(p)$ ).

Definition 0.7. Let $\mathbf{S}^{(n)}$ be the set of possibly infinitely-pp-definable connected subgroups of $\tilde{M}^{(n)}$-alternatively, members of $\mathbf{S}$ may be thought of as being the corresponding filter of subgroups (or formulas). Ordered by inclusion, $\mathbf{S}$ is essentially the stratified order of Poizat $[\mathbf{P o}]-$ a translation invariant version of the fundamental order $[\mathbf{L P}]$.

LEMma 0.8. Let $G$ be a possibily infinitely definable subgroup of $\tilde{M}^{(n)}$. Then $G \in \mathbf{S}$ if $G=G_{0}(p)$ for some $p \in S_{(n)}(0)$.

PROOF. $\Leftarrow$ This direction is 0.6 .

$\Rightarrow$ This is an easy exercise using 0.2 .

ThEOREM $0.9[\mathbf{P P}, \mathbf{Z g}]$. Let $p \in S(A), p \subseteq q \in S(B)$ where $B \supseteq A$. Then $q$ is a nonforking extension of $p$ iff $\mathcal{G}_{0}(p)=\mathcal{G}_{0}(q)$.

NOTE 0.10. (i) If $p \in S(M)$ with $M$ a model, then $\mathcal{G}_{0}(p)=\mathcal{G}(p)$.

(ii) If $\mathcal{G}_{0}(p)=\mathcal{G}(p)$ then $p$ is stationary (but not conversely-see [PP, Ro3]).

EXAMPLE 0.11 . Let $T=\operatorname{Th}\left(M_{0}=\mathbf{Z}_{4}^{\left(\aleph_{0}\right)}\right)$. Let $p \in S_{1}\left(M_{0}\right)$ be given by $p^{+}(x)=\langle x 2=0\rangle$ (where " \langle\rangle " denotes pp-deductive closure), and let $q \in S_{1}\left(M_{0}\right)$ be given by $q^{+}(x)=\langle(x-a) 2=0\rangle$ for some $a \in M_{0}$ of order 4 . Then $p$ is realized in $\mathbf{Z}_{4} \oplus M_{0}$ by the element $(2,0)$ of order 2 , and $q$ is realized by the element $(2, a)$ of order 4 .

We have $\mathcal{G}_{0}(p)=\mathcal{G}_{0}(q)=\langle v 2=0\rangle$-the corresponding group being that consisting of all elements of order 2 or 0 . From 0.9 , we see that $p$ is a nonforking extension of $p \nmid 0$, but $q$ is a forking extension of $q \nmid 0$. 
EXAMPLE 0.12 . Let $T=\operatorname{Th}\left(M_{0}=\mathbf{Z}_{4} \oplus Z_{2}^{\left(\aleph_{0}\right)}\right)$, and let $p \in S_{1}(0)$ be given by $p^{+}=\langle x=x\rangle$-the type of an element of order 4 . Note that $\mathcal{G}(p) \neq \mathcal{G}_{0}(p)$ : in fact $G(p)=\tilde{M}$, but $G_{0}(p)=\tilde{M} 2$, and $\left[G(p): G_{0}(p)\right]=2$. Nevertheless, one may easily see that $p$ is stationary (since the nongroup coset of $G_{0}(p)$ in $G(p)$ is definable). On the other hand, if we replace 2,4 by 3,9 throughout, then we obtain a type whose multiplicity (number of nonforking extensions to a model) is 2 .

EXAMPLE 0.13. Let $T=\operatorname{Th}\left(\mathbf{Z}_{(p)}\right)$, where $\mathbf{Z}_{(p)}$ is the localization of the module $\mathbf{Z}$ at the prime $p$. Let $p \in S_{1}(0)$ be given by $p^{+}=\langle x=x\rangle$. Then $G(p)=\tilde{M}$, but $\mathcal{G}_{0}(p)=\left\{p^{n} \mid x: n \in \omega\right\}$, and so $\left[G(p): G_{0}(p)\right]=\left[\tilde{M}: \bigcap\left\{\tilde{M} p^{n}: n \in \omega\right\}\right]$ is infinite.

One may check the multiplicity of $p, \operatorname{mult}(p)=2^{\aleph_{0}}$.

We will connect $\operatorname{mult}(p), G(p), G_{0}(p)$ in $\S 3$ below.

Now we go on to say something of the pure-injective models of $T$. It is convenient to work in the context of the monster model. So let $P(T)$ be the collection of all (necessarily pure-injective) direct summands of the monster model. When $M, N \in$ $\mathcal{P}(T)$ and we write $M \oplus N$ we will always be making the implicit assumptions that the sum $M+N$ is direct and is moreover pure in (so a direct summand of) $\tilde{M}$. We will make use of the following decomposition theorems.

THEOREM $0.14[\mathbf{F i}, \mathbf{Z g}]$. Let $N$ be a pure-injective module. Then $N$ has a decomposition of the sort $N=\overline{\bigoplus_{\lambda} N_{\lambda}} \bigoplus E$, where $N_{\lambda}$ is a direct-sum indecomposable pure injective and where $E$ has no nonzero indecomposable direct summands. Moreover, this decomposition is essentially unique (in any other decomposition the same factors occur, up to isomorphism, with the same multiplicity).

Ziegler $[\mathbf{Z g}]$ gives exact conditions on $T$ (when $|T|=\aleph_{0}$ ) such that the factor " $E$ " above never occurs. We will not need that here, but we will use his criterion for indecomposability (an expression of the fact that indecomposable pure injectives have local endomorphism rings). Say that a type $p \in S(0)$ is irreducible if its hull $N(p)$ is indecomposable.

THEOREM $0.15[\mathbf{Z g}]$. Let $p \in S(0)$. Then $p$ is irreducible iff, whenever $\psi_{1}, \psi_{2} \in p^{-}$there is $\varphi \in p^{+}$such that $\varphi \wedge \psi_{1}+\varphi \wedge \psi_{2} \in p^{-}$.

The situation in the totally transcendental case is particularly pleasant.

THEOREM $0.16[\mathbf{G a 2}]$. Let $M$ be a totally transcendental module. Then $M$ is pure injective and $M=\bigoplus_{\lambda} N_{\lambda}$, for suitable indecomposable direct summands $N_{\lambda}$ of $M$.

Moreover, Garavaglia showed that in the superstable case, the " $E$ " factor of 0.14 never occurs $[\mathbf{G a 3}]$. In such cases, the business of classifying the pure-injective (so in the t.t. case all) models reduces to describing how often each indecomposable direct summand may occur in a model (see $[\mathbf{P t 1}, \mathbf{Z g}]$ and below).

1. Unlimited types. Consider the example $M=\mathbf{Z}_{4} \oplus \mathbf{Z}_{2}^{\left(\aleph_{0}\right)}$ : the models of $T=\operatorname{Th}(M)$ are just those modules of the sort $\mathbf{Z}_{4} \oplus \mathbf{Z}_{2}^{(\kappa)}$ where $\kappa \geq \aleph_{0}$. So, for purposes of classifying and counting models of $T$, the component $\mathbf{Z}_{4}$ is not very relevant. We wish to be able to handle the type of situation illustrated by this example, and so we make the following definitions, which also facilitate an algebraic description of independence. 
DEFINITION 1.1. (a) If $N \in P(T)$, then $N$ is unlimited (over 0 ) if for every $M \vDash T, M \oplus N \vDash T$.

(b) If $p \in S(0)$, then $p$ is unlimited if $N(p)$ is unlimited.

(c) If $B, A \subseteq \tilde{M}$, then $B$ is unlimited over $A$ if, when we set $N(B \cup A)=N(A) \oplus N^{\prime}$ for some $N^{\prime}$, then $N^{\prime}$ is unlimited (note that this is well defined).

(d) A type or module which is not unlimited is limited.

The following propositions appear, in essence, elsewhere, so we content ourselves here with sketching the proofs.

PROPOSITION 1.2. (a) $N \in P(T)$ is unlimited iff there is some $M \vDash T$ with $M \oplus N \vDash T$, iff for every cardinal $\kappa$ and every $M \vDash T, M \oplus N^{(\kappa)} \vDash T$.

(b) $p \in S(0)$ is limited iff there are $\varphi \in p^{+}, \psi_{1}, \ldots, \psi_{n} \in p^{-}$with $T \vdash$ $\exists \bar{x}_{1}, \ldots, \bar{x}_{m} \forall \bar{x}\left(\varphi(\bar{x}) \rightarrow \bigvee_{i=1}^{n} \bigvee_{j=1}^{m} \psi_{i}\left(\bar{x}-\bar{x}_{j}\right)\right)$, where the $\psi_{i}$ may be taken so that the indices $\left[\varphi: \varphi \wedge \psi_{i}\right]$ are finite.

(c) $p \in S(0)$ is unlimited iff $\mathcal{G}(p)=\mathcal{G}_{0}(p)$, iff for every $\varphi \in p^{+}$and $\psi \in p^{-}$, $[\varphi: \varphi \wedge \psi]$ is infinite.

PROOF. (a) This is easy using the invariants.

(b) This is a direct compactness argument: one may write down a type, given any $M \vDash T$, whose realization implies a realization of $p$ "direct-sum independent" from $M$, and consistency of this type reduces to deciding sentences of the form shown.

(c) This is immediate from (b).

DEFinition 1.3. Suppose $A \in P(T)$. Then $B, C$ are direct-sum independent over $A$ if, for any pp formula $\varphi, \bar{b}$ in $B$ and $\bar{c}$ in $C$, if $\varphi(\bar{b}, \bar{c})$ holds then there is some $\bar{a}$ in $A$ with $\varphi(\bar{b}, \bar{a})$.

The algebraic significance of this is given by the following, which also explains the terminology.

Proposition 1.4. Let $A \in \mathcal{P}(T)$. Then $B$ and $C$ are direct-sum independent over $A$ if there is $N \in \mathcal{P}(T)$ of the form $N_{1} \oplus A \oplus N_{2}$ with $B \subseteq N_{1} \oplus A$ and $C \subseteq A \oplus N_{2}$.

PROOF. The direction $\Leftarrow$ is immediate from the fact that pp formulas behave well under projection.

The direction $\Rightarrow$ uses hulls. A proof may be found in Garavaglia [Ga4] (where the case dealt with is $A=0$, but the proof works equally well in this general case).

The connection between direct-sum (algebraic) independence and independence in the sense of nonforking is clearest when we deal with unlimited types.

COROLlary 1.5. Let $A \in P(T)$ and suppose that at least one of $B, C$ is unlimited over $A$. Then $B, C$ are independent over $A$ iff $B, C$ are direct-sum independent over $A$.

ProOF. This may be given a proof like that of 5.3 of $[\mathbf{P P}]$ where for the direction $\Rightarrow$ we use the obvious generalization of $1.2(\mathrm{c})$ : in particular, unlimited gives stationary, which is used in place of assuming the class of models is closed under direct sum.

Next, we give a more precise description of the behavior of limited types. 
Proposition 1.6. Let $p \in S(0)$ be limited. Then there is an integer $n$, such that any $N \in P(T)$, when decomposed according to 0.14 , contains at most $n$ copies of $N(p)$ and, if a model of $T$, contains exactly $n$ copies of $N(p)$, the latter provided $p$ is irreducible or $N$ sufficiently saturated.

PROOF. It will be sufficient to prove the latter statement. Note that in any case, by $1.2(\mathrm{c})$, we may choose $\varphi \in p^{+}, \psi \in p^{-}$with $\varphi>\psi,[\varphi: \psi]$ finite and such that there is no pp formula $\theta$ with $\varphi>\theta>\psi$. Then clearly, $n \leq$ $\operatorname{Inv}(T, \varphi, \psi) / \operatorname{Inv}(N(p), \varphi, \psi)$. The fact that we have equality here requires rather more work: see $[\mathbf{P t 1}]$ for the t.t. case and $[\mathbf{Z g}, 7.10]$ for the general case.

2. Ranks. Let $T$ denote a complete theory. We are concerned in this section with the analysis of certain ranks (on types and formulas) in the case of modules. The notion of forking is refined by the various stability ranks, which measure the degree of (in)dependence. There is no canonical rank, although they all measure forking, and they exist under more or less wide circumstances. We show here that, in the case of modules, there ranks coincide insofar as they are defined.

The ranks we are interested in are those of the form $R=R(-, \lambda)\left(\aleph_{0} \leq \lambda \leq \infty\right)$, in the notation of $[\mathbf{S h} \mathbf{1}]$, and the $U$-rank. As usual, we denote $R\left(-, \aleph_{0}\right)$ by MR (Morley rank) and $R(-, \infty)$ by $\mathrm{D}$ (Shelah degree). We show that in our case, for $p \in S(A), U(p)$ is the foundation rank of $G_{0}(p)$ in $\mathbf{S}$, and that for $R$ as above, if $R(p)<\infty$ then $U(p)=R(p)$. One consequence will be that in a superstable theory of modules, the $U$-rank is continuous.

We recall the definitions of these ranks. Let us emphasize that in this section all types are in only finitely many free variables.

DEFINITION 2.1. (i) ( $T$ stable) If $p \in S(A)$, the $U(p)>\alpha$ if $p$ has a forking extension $q$ with $U(q) \geq \alpha$, where $U(p) \geq 0$ provided $p$ is consistent (and $U$ is minimal subject to this).

(ii) $R(-, \lambda)$ is defined on formulas $\varphi(\bar{x}, \bar{a})$ with parameters. For $p \in S(A)$, one sets $R(p, \lambda)=\min \{R(\varphi, \lambda): \varphi \in p\}$, and $R(\varphi, \lambda) \geq \alpha$ if, for each $\beta<\alpha$ and each $\mu<\lambda$, there is some set $A$, and there are $q_{i} \in S(A)(i<\mu)$, such that: $\varphi \in q_{i}$ for each $i$, the $q_{i}$ are pairwise contradictory and $R\left(q_{i}, \lambda\right) \geq \beta$ for each $i$.

The ranks $R$, as defined on types, are continuous: that is, any $p \in S(A)$ has some $\varphi \in p$ such that whenever $\varphi \in q, R(q) \leq R(p)$.

FACT 2.2. ( $T$ stable) The $U$-rank has the property that if $U(p)=\alpha<\infty$, and if $\beta<\alpha$, then $p$ has an extension $q$ (necessarily forking) with $U(q)=\beta$. Moreover, $U$ is the least rank (among ranks satisfying certain axiomatic properties $[\mathbf{L s 1}]$ which are satisfied by the ranks $R(-, \lambda)$ ) with this property.

From now on, let $R=R(-, \lambda)$ for some $\lambda, \aleph_{0} \leq \lambda \leq \infty$.

FACTS 2.3. (i) $R\left(\bigvee_{1}^{n} \varphi_{i}\right)=\max \left\{R\left(\varphi_{i}\right): i=1, \ldots, n\right\}$.

(ii) $R(\varphi)=\alpha<\infty$ and $\beta<\alpha$ implies that there is some $\psi$ with $\psi \rightarrow \varphi$ and $R(\psi)=\beta$.

(iii) If $T$ has a group operation, then $R$ is preserved by translation.

(iv) $T$ is totally transcendental iff $\operatorname{MR}(x=x)<\infty$.

(v) $T$ if superstable iff $\mathrm{D}(x=x)<\infty$ iff $\mathrm{UR}(p)<\infty$ for all (1-) types (over any set).

Now, let us return to the context of modules. So $T$ now denotes a complete theory of $R$-modules, for some $R$. The theory $T$ is said to have the $d c c$ if there is no infinite 
descending chain of pp-definable subgroups in any (so every) model of $T$. $T$ is said to have the superstable $d c c$ if $T$ has no infinite descending chain of pp-definable subgroups, each of infinite index in the preceding one (in some, equivalently in every, model). It makes sense to relativize the dcc to a pp-definable subgroup $\varphi$, by considering only those pp-definable subgroups contained in $\varphi$ : the lattice of such subgroups is a sublattice of that consisting of all pp-definable subgroups. Analogously to the global case $(\varphi(x)=" x=x ")$ [Ga1] one obtains the following.

Proposition 2.4. Suppose $\varphi(\bar{x}, \bar{a})$ is $p p$. Then:

(a) $\operatorname{MR}(\varphi(\bar{x}, \bar{a}))<\infty$ iff $\varphi(\bar{x}, \overline{0})$ has the dcc;

(b) $\mathrm{D}(\varphi(\bar{x}, \bar{a}))<\infty$ iff $\varphi(\bar{x}, \overline{0})$ has the superstable dcc.

We want first to show that the ranks $R$ depend only on pp formulas. It is enough to show the following. We are grateful to Simon Thomas for pointing out a simple proof.

LEMMA 2.5. Let $\chi(\bar{x})=\varphi(\bar{x}, \bar{a}) \wedge \bigwedge_{1}^{n} \neg \psi_{i}\left(\bar{x}, \bar{a}_{i}\right)$ where $\varphi, \psi_{1}, \ldots, \psi_{n}$ are $p p$, and $\chi$ is consistent. Then $R(\chi)=R(\varphi)$.

ProOF. This is by induction on $n$, the case $n=0$ being trivial. So assume the truth of the result for $n-1$. Then we may certainly assume that $\chi \leftrightarrow \varphi \wedge$ $\bigwedge_{i=1 ; i \neq j}^{n} \neg \psi_{i}$ for each $j$. Moreover, we may suppose $\varphi(\bar{x}, \overline{0}) \geq \psi_{i}(\bar{x}, \overline{0})$ for each $i$.

Choose $i \leq n$. Since $\chi$ is consistent, there is some coset $\psi_{i}\left(\bar{x}-\bar{c}_{i}, \overline{0}\right)$ of $\psi_{i}(\bar{x}, \overline{0})$ such that $\psi_{i}\left(\bar{x}-\bar{c}_{i}, \overline{0}\right) \subseteq \varphi(\bar{x}, \bar{a})$, and such that $\theta(\bar{x})=\psi_{i}\left(\bar{x}-\bar{c}_{i}, \overline{0}\right) \wedge \bigwedge_{j=1}^{n} \neg \psi_{j}\left(\bar{x}, \bar{a}_{j}\right)$ is consistent. In particular, $\psi_{i}\left(\bar{x}-\bar{c}_{i}, \overline{0}\right) \cap \psi_{i}\left(\bar{x}, \bar{a}_{i}\right)=\varnothing$. Therefore, $\theta(\bar{x})$ is equivalent to the formula $\psi_{i}\left(\bar{x}-\bar{c}_{i}, \overline{0}\right) \wedge \bigwedge_{j=1 ; j \neq i}^{n} \neg \psi_{j}\left(\bar{x}, \bar{a}_{j}\right)$.

Now, $\theta(\bar{x}) \rightarrow \chi(\bar{x})$, so certainly $R(\theta) \leq R(\chi)=\alpha$ say. By the induction hypothesis, $R\left(\psi_{i}\left(\bar{x}-\bar{c}_{i}, \overline{0}\right)\right) \leq \alpha$, so by (iii) above, $R\left(\psi_{i}\left(\bar{x}, \bar{a}_{i}\right)\right) \leq \alpha$, and this for all $i \leq n$.

Note that $\varphi(\bar{x}, \bar{a}) \leftrightarrow \chi(\bar{x}) \vee \bigvee_{i=1}^{n} \psi_{i}\left(\bar{x}, \bar{a}_{i}\right)$, so by (i) above, $R(\varphi(\bar{x}, \bar{a}))=\alpha$, as required.

From pp-elimination of quantifiers we therefore obtain the following.

Corollary 2.6. Let $p \in S(A)$. Then there is some $\varphi \in p^{+}$with $R(\varphi)=$ $R(p)$.

We have the further corollary.

Corollary 2.7. Let $\varphi(\bar{x}, \bar{a})$ be $p p$, with $R(\varphi)=\alpha<\infty$. Let $\beta<\alpha$. Then there is some pp formula $\psi(\bar{x}, \bar{b})$ with $\psi(\bar{x}, \bar{b}) \rightarrow \varphi(\bar{x}, \bar{a})$ and with $R(\psi(\bar{x}, \bar{b}))=\beta$.

PROOF. First we have by 2.3(ii) and 0.1 that there is some formula $\chi(\bar{x})$ of the form $\psi(\bar{x}, \bar{b}) \wedge \bigwedge_{1}^{n} \neg \psi_{i}\left(\bar{x}, \bar{b}_{i}\right)$ with $\psi, \psi_{1}, \ldots, \psi_{n}$ pp, such that $\chi(\bar{x}) \rightarrow \varphi(\bar{x}, \bar{a})$ and $R(\chi)=\beta$. Replacing $\psi(\bar{x}, \bar{b})$ by $\psi(\bar{x}, \bar{b}) \wedge \varphi(\bar{x}, \bar{a})$, we may assume $\psi(\bar{x}, \bar{b}) \rightarrow \varphi(\bar{x}, \bar{a})$. Then with 2.5 we finish.

In order to describe the ranks more precisely, we need some more information connecting types and members of $\mathbf{S}$. What we are going to show is that the $U$-rank of a type $p$ is the foundation rank of $G_{0}(p)$ in $\mathbf{S}$. The next result obviously fills the only remaining gap in establishing the connection between $\mathbf{S}$ and forking. 
Proposition 2.8. Let $p \in S(A)$, and let $H \in \mathbf{S}$ be such that $H \leq G_{0}(p)$. Let $\bar{c}$ realize $p$. Then there is $q \in S(\bar{c})$ with $G(q)=G_{0}(q)=H$ such that the unique nonforking extension of $q$ to $A^{-} \bar{c}$ extends $p$. In particular, there is $q^{\prime} \supseteq p$ with $G\left(q^{\prime}\right)=H$.

Proof. Let $H=\bigcap \mathcal{G}$. Let $q=\{\varphi(\bar{x}-\bar{c}): \varphi(\bar{x}) \in \mathcal{G}\} \cup\{\neg \psi(\bar{x}, \bar{c}): \psi$ is pp, $\psi(\bar{x}, \overline{0}) \notin \mathcal{G}\}$. By $0.2, q$ is consistent, and clearly $G(q)=H$, and so $q$ is stationary. Let $(0.8) q_{0} \in S(0)$ be such that $G\left(q_{0}\right)=H$.

Let $q^{\prime}$ be the nonforking extension of $q$ to $A \frown \bar{c}$. We claim that $q^{\prime}$ extends $p$. It will be enough to show that $\left(q^{\prime} \uparrow A\right)^{+}=p^{+}$.

Let $\varphi(\bar{x}, \bar{a}) \in p^{+}$- so $\varphi(\bar{c}, \bar{a})$, and also $\varphi(\bar{x}, \bar{a}) \in \mathcal{G}(p)$, so $\varphi(\tilde{M}, \overline{0}) \geq H$. Therefore, by construction, $\varphi(\bar{x}, \overline{0}) \in q_{0}$, and $\varphi(\bar{x}-\bar{c}, \overline{0}) \in q \subset q^{\prime}$. Together with $\varphi(\bar{c}, \bar{a})$, this yields $\varphi(\bar{x}, \bar{a}) \in q^{\prime}$ - that is $\varphi(\bar{x}, \bar{a}) \in\left(q^{\prime} \uparrow A\right)^{+}$.

Conversely, if $\varphi(\bar{x}, \bar{a}) \in\left(q^{\prime} \uparrow A\right)^{+} \subseteq q^{\prime}$ then, since therefore $\varphi(\bar{x}, \overline{0}) \in \mathcal{G}\left(q^{\prime}\right)=$ $\mathcal{G}(q)=\mathcal{G}\left(q_{0}\right)$, one has $\varphi(\bar{x}, \overline{0}) \in q_{0}$. So, by construction, we have $\varphi(\bar{x}-\bar{c}, \overline{0}) \in$ $q \subseteq q^{\prime}$. Combining with $\varphi(\bar{x}, \bar{a}) \in q^{\prime}$ we obtain $\varphi(\bar{c}, \bar{a})$. Hence $\varphi(\bar{x}, \bar{a}) \in p^{+}$, as required.

Corollary 2.9. Let $p \in S(A)$ and suppose $H \in \mathbf{S}$. Then $p$ has a forking extension $q$ with $G_{0}(q)=H$ iff $H<G_{0}(p)$.

COROLlary 2.10. Let $p \in S_{(n)}(A)$. Then $U(p)$ is the foundation rank of $G_{0}(p)$ in $\mathbf{S}^{(n)}$.

Proposition 2.11. Let $p \in S(A)$ and suppose $R(p)<\infty$. Then $R(p)=U(p)$.

Proof. It is known that, in general, $U(p) \leq R(p)$. The proof that $U(p)=R(p)$ is by induction on $R(p)$.

So suppose $R(p)=\alpha$. Let $\beta<\alpha$. By 2.6 there is $\varphi(\bar{x}, \bar{a}) \in p^{+}$with $R(\varphi)=\alpha$. By 2.7 choose $\psi(\bar{x}, \bar{b})$ pp with $\psi(\bar{x}, \bar{b}) \rightarrow \psi(\bar{x}, \bar{a})$ and $R(\psi)=\beta$.

Let $H$ be the connected component of the group $\psi(\tilde{M}, \overline{0}): H=G_{0}(\psi(\bar{x}, \overline{0}))=$ $\bigcap\{\varphi$ pp: $[\psi: \psi \wedge \varphi]$ finite $\}$. Noting that (by property (ii)) the index $[\varphi(\bar{x}, \overline{0})$ : $\psi(\bar{x}, \overline{0})]$ is infinite, we have $H<G_{0}(p)$.

So by 2.9 there is a forking extension $q$ of $p$ with $G_{0}(q)=H$. Observing that, for $\psi^{\prime} \in \mathcal{G}_{0}(q), R\left(\psi^{\prime}\right) \geq R(\psi)=\beta$ (since $\psi$ "generates" $H$ ), we have $R(q) \geq \beta$, and hence (since $\psi \in \mathcal{G}_{0}(q)$ and by (i)), $R(q)=\beta$. So by the induction hypothesis, $U(q)=\beta$.

Thus $U(p) \geq \alpha$. Since in any case $U(p) \leq R(p)=\alpha$, we are finished.

It is clear, by 2.4 and the fact that $\lambda \geq \mu$ implies $R(\varphi, \lambda) \leq R(\varphi, \mu)$, that if $\varphi(\bar{x}, \bar{a})$ is pp and $R(\varphi)<\infty$ then $\varphi(\bar{x}, \overline{0})$ has the superstable dcc. Thus, if $R(\varphi)<$ $\infty$, we may define a suitable foundation rank $U^{\prime}$ on the pp-definable subgroups contained in $\varphi(\tilde{M}, \overline{0}): U^{\prime}(\psi) \geq \alpha+1$ if $\psi$ contains some $\psi^{\prime}$ of infinite index with $U^{\prime}\left(\psi^{\prime}\right) \geq \alpha ; U^{\prime}(0)=0 ; U^{\prime}(\psi) \geq \lambda$ if $U^{\prime}(\psi)>\alpha$ for all $\alpha<\lambda$.

Given $\varphi(\bar{x}, \bar{a})$ pp, let $\mathcal{G}_{0}(\varphi)$ be the set $\{\psi(\bar{x}): \psi$ is pp and $[\varphi(\bar{x}, \overline{0}): \varphi(\bar{x}, \overline{0}) \wedge \psi(\bar{x})]$ is finite $\}$ generated by $\varphi(\bar{x}, \overline{0})$. Let $G_{0}(\varphi)=\bigcap \mathcal{G}_{0}(\varphi)$.

COROllary 2.12. Let $\varphi(\bar{x}, \bar{a})$ be $p p$ with $R(\varphi)<\infty$. Then $R(\varphi)=U^{\prime}(\varphi(\bar{x}, \overline{0}))$ $=$ the foundation rank of $G_{0}(\varphi)$ in $\mathbf{S}$. If $\varphi(\bar{x}, \bar{a}) \in p$ and $R(\varphi)=R(p)$, then this common value is also $U(p)$. 
COROLLARY 2.13. If $T$ is superstable, then the $U$-rank is continuous. That is if $p \in S(A)$ then there is some $\varphi(\bar{x}, \bar{a}) \in p^{+}$with $U(p)=\sup \left\{U\left(p^{\prime}\right): \varphi(\bar{x}, \bar{a}) \in\right.$ $\left.p^{\prime}\right\}$.

COROLlaRY 2.14. If $M \prec_{1}^{+} M^{\prime}$ and $\varphi$ is pp then $R^{M}(\varphi) \leq R^{M^{\prime}}(\varphi)$.

EXAMPLE 2.15. For any $n \in \omega$, there is an abelian group $M$ with $\operatorname{MR}(M)=n$ : let $M=\mathbf{Z}_{p^{n}}^{\left(\aleph_{0}\right)}$ where $p$ is any prime (so $\mathbf{S}^{1}$ is a chain of length $n$ ).

EXAMPLE 2.16. Let $p$ be a prime. Then $\operatorname{MR}\left(\mathbf{Z}_{p^{\infty}}^{\left(\aleph_{0}\right)}\right)=\omega$ (where $\mathbf{Z}_{p^{\infty}}$ is the indecomposable, divisible $p$-torsion group). One may check that $\operatorname{MR}\left(\bigoplus_{p \text { prime }} \mathbf{Z}_{p^{\infty}}^{\left(\aleph_{0}\right)}\right)$ $=\omega$ still. Since every totallytranscendental abelian group has the form $\bigoplus_{p \text { prime }} \mathbf{Z}_{p^{\infty}}^{\left(\kappa_{p}\right)} \oplus \mathbf{Q}^{(\kappa)} \oplus B$ for some $\kappa_{p}, \kappa$, where $B$ has bounded exponent (so has finite Morley rank) $[\mathbf{M c}]$, it follows without much difficulty that, for any abelian $\operatorname{group} M, \operatorname{MR}(M)<\omega+\omega$ if $\operatorname{MR}(M)<\infty[$ Ro1].

EXAMPLE 2.17. Let $M=\mathbf{Z}_{(p)}$. The only type in $S_{1}(0)$ with $\mathrm{MR}<\infty$ is the trivial type " $x=0$ ". The type $p \in \mathbf{S}_{1}(0)$ given by $p^{+}(x)=\left\{p^{n} \mid x: n \in \omega\right\}$ has $U(p)=1$ and is stationary. Since, clearly $\left|\mathbf{S}^{\prime}\right|=2$, every other type $q \in S_{1}(0)$ has $\mathcal{G}_{0}(q)=\mathcal{G}(p)$, and has $U(q)=1$. Hence $U(T)\left(=\sup \left\{U(q): q \in S_{1}(0)\right\}\right)=1$.

EXAMPLE 2.18. Let $M=\mathbf{Z}_{4} \oplus \mathbf{Z}_{2}^{\left(\aleph_{0}\right)}$. There are four types in $S_{1}(0)$, given by $p_{0}^{+}(x)=\langle x=0\rangle ; p_{1}^{+}(x)=\langle x 2=0 \wedge 2 \mid x\rangle ; p_{2}^{+}(x)=\langle x 2=0\rangle ; p_{3}^{+}(x)=\langle x=x\rangle$. We have $\mathfrak{G}_{0}\left(p_{3}\right)=\mathcal{G}\left(p_{2}\right)=\mathcal{G}_{0}\left(p_{2}\right)=\mathcal{G}_{0}\left(p_{1}\right)=\mathcal{G}\left(p_{0}\right)=\mathcal{G}_{0}\left(p_{0}\right)$, and so $\operatorname{MR}(M)=1$.

On the other hand it is clear that $\operatorname{MR}\left(M^{\left(\aleph_{0}\right)}\right)=3$.

EXAMPLE 2.19. Since every pp-definable subgroup of a ring is a left ideal, and finitely generated left ideals are (right) pp-definable, we have:

if $R$ is left artinian then $M R(R) \leq \operatorname{MR}\left(R^{\left(\aleph_{0}\right)}\right)=$ length of $\left({ }_{R} R\right)$, which is finite.

One should note that if $R$ is left coherent (e.g., if $R$ is left noetherian), then the pp-definable subgroups of $R_{R}$ are precisely the finitely generated left ideals [Ro2, $\mathrm{Zm}$.

In general, since every projective module is a direct summand of a sum of copies of $R, 2.14$ yields $\operatorname{MR}(P) \leq \operatorname{MR}\left(R^{\left(\aleph_{0}\right)}\right)$ for any projective module $P$.

Finally in this section, we point out an easy fact for modules which is useful when computing ranks, and which we will apply in $\S 6$. First we set up some notation (making the useful confusion of formulas with the sets they define).

Let $\psi^{\prime}(y)$ and $\psi(y, x)$ be pp formulas over 0 . Let $\varphi(x)$ be $\exists y\left(\psi^{\prime}(y) \wedge \psi(y, x)\right)$ (the projection of $\bigcup\left\{\psi(y, x): y \in \psi^{\prime}\right\}$ ), where we suppose moreover that $\psi^{\prime}(y) \rightarrow$ $\exists x \psi(y, x)$. So $\varphi(x)$ is a pp-definable subgroup which is the union of cosets of $\psi(0, x)$, indexed by $\psi^{\prime}(y)$.

Now let $\theta(x)$ be pp with $\theta(x) \subseteq \varphi(x)$. Then clearly

$$
\theta(x) \leftrightarrow \exists y\left(\psi^{\prime}(y) \wedge \psi(y, x) \wedge \theta(x)\right) \leftrightarrow \exists y\left(\chi^{\prime}(y) \wedge \chi(y, x)\right),
$$

where $\chi(y, x)$ is $\psi(y, x) \wedge \theta(x)$ and $\chi^{\prime}(y)$ is $\psi^{\prime}(y) \wedge \exists x(\chi(y, x))$. Notice that $\chi, \chi^{\prime}$ are pp; that $\chi^{\prime}(y) \subseteq \psi^{\prime}(y)$; and that $\chi(0, x) \subseteq \psi(0, x)$.

Then one easily obtains, retaining the above notation, the following.

PROPOSITION 2.20. (i) $\theta(x) \subset \varphi(x)$ iff either $\chi(0, x) \subset \psi(0, x)$ or $\chi^{\prime}(y) \subset$ $\psi^{\prime}(y)$. 
(ii) $[\varphi: \theta]$ is infinite iff either $[\psi(0, x): \chi(0, x)]$ or $\left[\psi^{\prime}(y): \chi^{\prime}(y)\right]$ is infinite.

The following consequences of this (still retaining the same notation) are left to the reader.

COROLlaRY 2.21. (i) $\varphi(x)$ has the (superstable) dcc iff both $\psi(0, x)$ and $\psi^{\prime}(y)$ have the (superstable) dcc.

(ii) Moreover, if $R(\psi(0, x))=\alpha$ and $R\left(\psi^{\prime}(y)\right)=\beta(\alpha, \beta<\infty)$ then $R(\varphi(x)) \leq$ $\alpha \oplus \beta$.

3. Nonmultidimensionality, strong types and multiplicity. In this section we consider briefly several topics.

First we define the "free part" of a type.

Definition 3.1. Let $p \in S(A)$. Denote by $p_{*}$ the unique type over 0 such that $\left(p_{*}\right)^{+}=\mathcal{G}_{0}(p)$. Note that such a type exists by 0.8 and, by $1.2(\mathrm{c}), p_{*}$ is unlimited.

Proposition 3.2 (CF. [Pt2]). Let $p \in S(M)$ with $M \vDash T$, and suppose $\bar{a}$ in $M$ satisfies $p^{+}$(though not necessarily $\left.p\right)$. Then:

(i) $p$ is definable over $\bar{a}$;

(ii) if $\bar{b}$ realizes $p$, then $\bar{b}-\bar{a}$ realizes $p_{*}$ and, moreover, $\bar{b}-\bar{a}$ and $M$ are independent over 0 ;

(iii) if $\bar{c}$ realizes $p_{*}$, and if $\bar{c}$ and $M$ are independent over 0 , then $\operatorname{tp}(\bar{a}+\bar{c} / M)=p$.

ProOF. (i) For this, it suffices by 0.1 to note that, for any pp formula $\varphi(\bar{x}, \bar{y})$ which is represented in $p(\bar{x})$, we have, for all $\bar{m}$ in $M$, that $\varphi(\bar{x}, \bar{m}) \in p$ iff $M \vDash$ $\varphi(\bar{a}, \bar{m})$.

(ii) Since $\bar{a}$ is in $M, \operatorname{tp}(\bar{b}-\bar{a} / M)$ is a translate, over $M$ of $\operatorname{tp}(\bar{b} / M)$, and hence $\mathcal{G}(\operatorname{tp}(\bar{b}-\bar{a} / M))=\mathcal{G}(p)$.

On the other hand, if $\varphi \in \mathcal{G}(p)$ then, since $M$ is a model, we have $\varphi\left(\bar{x}-\bar{a}^{\prime}\right) \in p^{(+)}$ for some $\bar{a}^{\prime}$ in $M$. Hence $\varphi\left(\bar{a}-\bar{a}^{\prime}\right)$ and $\varphi\left(\bar{b}-\bar{a}^{\prime}\right)$ hold. Therefore we have $\varphi(\bar{b}-\bar{a})$, and so $\mathcal{G}(p) \subseteq \operatorname{tp}(\bar{b}-\bar{a} / 0)$.

Therefore $\mathcal{G}_{0}(p)=\mathcal{G}(p)=\operatorname{tp}(\bar{b}-\bar{a} / 0)=\mathcal{G}(\operatorname{tp}(\bar{b}-\bar{a} / M))$. So $\bar{b}-\bar{a}$ realizes $p_{*}$, and is independent from $M$ over 0 (by 0.9 ).

(iii) If $\varphi \in \mathcal{G}(p)$, then $\varphi(\bar{c})$ holds and, for some $\bar{a}^{\prime}$ in $M, \varphi\left(\bar{a}-\bar{a}^{\prime}\right)$ holds. Hence $\varphi\left(\bar{a}+\bar{c}-\bar{a}^{\prime}\right)$ holds, whence $\varphi \in \mathcal{G}(\operatorname{tp}(\bar{a}+\bar{c} / M))$. Conversely, if $\varphi \in \mathcal{G}(\operatorname{tp}(\bar{a}+\bar{c} / M))$, say $\bar{a}^{\prime \prime}$ is in $M$, with $\varphi\left(\bar{a}+\bar{c}-\bar{a}^{\prime \prime}\right)$ then, since $\bar{c}$ and $M$ are independent over 0 , we have (by 0.9$), \varphi(\bar{c})$. Therefore $\varphi \in\left(p_{*}\right)^{+}=\mathcal{G}(p)$.

So $p$ and $\operatorname{tp}(\bar{a}+\bar{c} / M)$ represent the same pp formulas. Furthermore, $\varphi(\bar{x}, \bar{m}) \in p^{+}$ implies $\varphi(\bar{c}, \overline{0})$ and $\varphi(\bar{a}, \bar{m})$, hence $\varphi(\bar{a}+\bar{c}, \bar{m})$. Therefore $\operatorname{tp}(\bar{a}+\bar{c} / M)=p$.

COROLlary 3.3. Let $p \in S(M)$ where $M$ is a pure-injective model. Then:

(i) $p$ is definable over a finite tuple;

(ii) some $M$-translate of $p$ is definable over 0 .

Proof. Since $M$ is pure-injective, $p^{+}$is satisfied in $M$. The conclusions now follow by (i), (ii) of 3.2 .

COROLlaRY 3.4. Let $M$ be a pure-injective model, $p \in S(M), f$ an automorphism of $M$, and let $p^{\prime}=f(p)$. Then for any $N \succ M, p$ is realized in $N$ iff $p^{\prime}$ is realized in $N$.

ProOF. Actually, all we need is that $M \subseteq N \subseteq \tilde{M}$ and $N$ is a group. 
Since $M$ is pure injective, let $\bar{a}$ in $M$ satisfy $p^{+}$, and let $\bar{a}^{\prime}$ satisfy $\left(p^{\prime}\right)^{+}$. Note that $\mathcal{G}(p)=\mathcal{G}\left(p^{\prime}\right)$. By 3.2 (ii) then (iii), it follows that $\bar{b}$ realized $p$ iff $\bar{b}-\bar{a}+\bar{a}^{\prime}$ realizes $p^{\prime}$.

COROLlary 3.5. Any theory of modules in nonmultidimensional. (Note that we do not assume superstability here.)

Proof. This is by 3.4, using the definition in [Sh1, Definition V 5.2].

Now let $p \in S(A)$. The multiplicity, $\operatorname{mult}(p)$, of $p$ is the number of nonforking extensions of $p$ to any model (this is well defined), and $p$ is stationary if mult $(p)=1$. We already noted in $\S 0$ that if $\mathcal{G}(p)=\mathcal{G}_{0}(p)$ then $p$ is stationary, but that the converse is false. The precise determination of those types which are stationary, even in the abelian group case, is not straightforward (see $[\mathbf{R o 3}]$ ), and so we will be content here to establish a bound on $\operatorname{mult}(p)$ in terms of $\mathcal{G}_{(0)}(p)$.

Recall that if $\bar{c}, \bar{d}$ are tuples of the same length, then they have the same strong type, $\operatorname{stp}(\bar{c} / A)=\operatorname{stp}(\bar{d} / A)$, if $E(\bar{c}, \bar{d})$ holds whenever $E\left(\bar{x}_{1}, \bar{x}_{2}\right)$ is an $A$-definable equivalence with only finitely many classes. It is known that for $p \in S(A), \operatorname{mult}(p)$ is equal to the number of strong types over $A$ extending $p$.

Proposition 3.6 below shows, in effect, that (in the case of modules) the only finite equivalence relations needed to determine $\operatorname{stp}(\bar{c} / A)$ are those of the form:

$$
E\left(\bar{x}_{1}, \bar{x}_{2}\right)=\left(\neg \varphi\left(\bar{x}_{1}, \bar{a}\right) \wedge \neg \varphi\left(\bar{x}_{2}, \bar{a}\right)\right) \vee\left(\varphi\left(\bar{x}_{1}, \bar{a}\right) \wedge \varphi\left(\bar{x}_{2}, \bar{a}\right) \wedge \psi\left(\bar{x}_{1}-\bar{x}_{2}\right)\right),
$$

where $\varphi, \psi$ are pp, $\psi(\bar{x}) \rightarrow \varphi(\bar{x}, \overline{0}),[\varphi(\bar{x}, \overline{0}): \psi(\bar{x})]$ is finite, and $\varphi(\bar{x}, \bar{a}) \in \operatorname{tp}(\bar{c} / A)$.

Proposition 3.6. Let $\operatorname{tp}(\bar{c} / A)=\operatorname{tp}(\bar{d} / A)=p$. Then $\operatorname{stp}(\bar{c} / A)=\operatorname{stp}(\bar{d} / A)$ iff $\psi(\bar{c}-\bar{d})$ holds whenever $\psi(\bar{x}) \in \mathcal{G}_{0}(p)$.

PROOF. $\Rightarrow$ This is clear (using the equivalence relation $E$ above for suitable $\varphi(\bar{x}, \bar{a}))$.

$\Leftarrow$ Let $M \supseteq A$ be a model such that $\operatorname{tp}(\bar{c} / M)$ and $\operatorname{tp}(\bar{d} / M)$ do not fork over $A$. If $\operatorname{stp}(\bar{c} / A) \neq \operatorname{stp}(\bar{d} / A)$, then clearly $\operatorname{tp}(\bar{c} / M) \neq \operatorname{tp}(\bar{d} / M)$. Say $\psi(\bar{x}, \bar{b})$ is pp with $\bar{b}$ in $M$ with $\psi(\bar{c}, \bar{b}) \wedge \neg \psi(\bar{d}, \bar{b})$. Since the types of $\bar{c}, \bar{d}$ over $M$ are nonforking over $A$, we have $\psi(\bar{x}, \overline{0}) \in \mathcal{G}_{0}(p)$, and also $\neg \psi(\bar{c}-\bar{d}, \overline{0})$ holds.

COROllary 3.7. Let $p \in S(A)$.

(i) If $\left[G(p): G_{0}(p)\right]$ is finite, then $\operatorname{mult}(p) \leq\left[G(p): G_{0}(p)\right]$.

(ii) If $\left[G(p): G_{0}(p)\right]$ is infinite, then $2^{\aleph_{0}} \leq \operatorname{mult}(p) \leq|T|^{\aleph_{0}}$.

PROOF. The only part which now requires proof is the lower bound in part (ii). Let $\theta(\bar{v})$ be a pp formula in $\mathcal{G}_{0}(p) \backslash \mathcal{G}(p)$, and let $C$ be a coset of $\theta$ which is contained in (the set defined by) $p$. Consider the tree consisting of those cosets contained in $C$ which are cosets of formulas in $\mathcal{G}_{0}(p)$. Observe that since the index $\left[\theta: \mathcal{G}_{0}(p)\right]$ is infinite this tree has infinite depth. Now consider the subtree $T$ consisting of those cosets which are not definable over $A$. To deduce the existence of $2^{\aleph_{0}}$ strong type extensions of $p$ it will be sufficient to show that this latter tree is cofinally branching.

Let $C^{\prime} \in T$ be a coset of (say) $\varphi \in \mathcal{G}_{0}(p)$ : let $\psi \in \mathcal{G}_{0}(p)$ be strictly contained in $\varphi$. Supposing for a contradiction that there is at most one coset of $\psi$ in $C^{\prime}$ which is undefinable over $A$, there must be a definable one, say $\psi^{\prime}(\bar{v}, \bar{b})$, where $\bar{b}$ is in $A$ and $\psi^{\prime}(\bar{v}, \overline{0}) \leftrightarrow \psi(\bar{v})$. Then the formula $\exists \bar{w}\left(\psi^{\prime}(\bar{w}, \bar{b}) \wedge \varphi(\bar{v}-\bar{w})\right)$ defines $C$-contradiction as required. 
The fact that we do not have equality in 3.7(i) is due to the fact that, although for each $\bar{m}$ in a model, satisfying $p^{+}, p^{+}(\bar{x}) \cup\left\{\varphi(\bar{x}-\bar{m}): \varphi \in \mathcal{G}_{0}(p)\right\}$ is consistent, it need not be the case that $p(\bar{x}) \cup\left\{\varphi(\bar{x}-\bar{m}): \varphi \in \mathcal{G}_{0}(p)\right\}$ is consistent (see 0.12 ). Examples 0.11-0.13 illustrate 3.7.

4. Saturation and pure-injective models. Saturation in modules can be resolved into "vertical" and "horizontal" components. The vertical component is pure injectivity - positive saturation. The horizontal component is fatness (in the sense of $[\mathbf{S g 2}])$. In this section we make this claim precise (4.5) and also consider homogeneity. Note that $|T|=|R|+\aleph_{0}$. It is clear that if $M$ is $|T|^{+}$-saturated then $M$ is pure injective.

Proposition 4.1. Let $\kappa \geq|T|^{+}$. Let $M$ be $\kappa$-saturated and suppose $N \prec M$ is pure injective. Then $N$ also is $\kappa$-saturated.

Proof. So let $A \subseteq N$ with $|A|<\kappa$ and take $p \in S_{1}(A)$. Without loss of generality we may suppose $A=M^{\prime} \prec N$. Since $N$ is pure injective there is $c \in N$ satisfying $p^{+}$.

Let $B \subseteq M$ be such that $\operatorname{tp}\left(c^{-} M^{\prime} / M\right)$ is definable over $B$, in the strong sense that if $\psi\left(c, \bar{m}^{\prime}, \bar{m}\right)$ is pp with $\bar{m}^{\prime}$ in $\bar{M}^{\prime}$ and $\bar{m}$ in $M$, then there is $\bar{b}$ in $B$ with $\psi\left(c, \bar{m}^{\prime}, \bar{b}\right)$. Clearly $B$ may be chosen with $|B|<\kappa$. Since $M$ is $\kappa$-saturated take $d \in M$ realizing the nonforking extension of $p_{*}$ to $B$. We claim that $\operatorname{tp}\left(c+d / M^{\prime}\right)=p$.

Suppose $\varphi(x, \bar{a}) \in p^{+}$. So we have $\varphi(c, \bar{a})$ and $\varphi(d, \overline{0})$, whence $\varphi(c+d, \bar{a})$.

Conversely, suppose $\varphi(c+d, \bar{a})$. By choice of $B$, there is $b \in B$ with $\varphi(c+d, \bar{a})$. Therefore $\varphi(d-b, \overline{0})$. Since $d$ realizes $p_{*}^{B}, d$ and $b$ are independent over 0 , hence $(\S 1) \varphi(x, \overline{0}) \in p_{*}$, and so $\varphi(d, \overline{0})$. Therefore $\varphi(c, \bar{a})$ holds, whence $\varphi(x, \bar{a}) \in p$.

PROPOSITION 4.2. Let $T$ be superstable. Then any elementary extension of a pure-injective model is pure injective.

Proof. Say $M \succ N$, where $N$ is superstable and pure injective. Set $M=N \oplus K$. It is clear on considering the invariants that $K$ is totally transcendental (note that $K$ is unlimited). Hence $K$ is pure injective $(0.16)$, whence $M$ is pure injective.

LEMMA 4.3. Let $T$ be superstable. Then any pure-injective model of $T$ is $\aleph_{0}$ homogeneous.

Proof. Say $N \vDash T$ is pure-injective. Suppose $\bar{a}, \bar{b}$ are in $N$ with $\operatorname{tp}(\bar{a})=\operatorname{tp}(\bar{b})$. Set $N=N^{\top}(\bar{a}) \oplus N_{1}=N(\bar{b}) \oplus N_{2}$. By the basic properties of hulls, there is an isomorphism $f: N(\bar{a}) \rightarrow N(\bar{b})$ taking $\bar{a}$ to $\bar{b}$.

Now, $N$ and its direct summands, have essentially unique decomposition as pureinjective hulls of direct sums of indecomposable summands. Note that, although when we decompose $N(a), N(b)$ we may obtain infinitely many summands (e.g. $a=$ 1 in $\mathbf{Z}$ ), each summand occurs only finitely many times in the decomposition. For otherwise, say $\overline{N_{0}^{\left(\aleph_{0}\right)}}$ is a direct summand of $N(\bar{a})$. Then clearly $N_{0}$ is unlimited. Choose a nonzero element $a_{1}$ in each copy of $N_{0}$. So we have an infinite independent family $\left\{a_{1}: i \in \omega\right\}$ such that each member is dependent over 0 on the finite tuple $\bar{a}$. This contradicts superstability.

Since each summand of $N(\bar{a}) \simeq N(\bar{b})$ occurs only finitely many times, it follows that $N_{1} \simeq N_{2}$ (since the decomposition of $N$ is essentially unique), say $g: N_{1} \cong N_{2}$. Then $f+g$ is an automorphism of $N$, taking $\bar{a}$ to $\bar{b}$, as required. 
It is worth pointing out that at least if $T$ is countable we need the full strength of superstability of $T$ in 4.3 (of course, even if $T$ is nonsuperstable there will be certain pure-injective $N \vDash T$ which are $\aleph_{0}$-homogeneous). If $T$ is not superstable then it easily follows (see 7.4 below) that there is some unlimited $N \in \mathcal{P}(T)$ which is not totally transcendental. So we may find pp formulas $\varphi_{i}$, with $\varphi_{0}(N)>$ $\varphi_{1}(N)>\cdots$. Choose $a_{i} \in \varphi_{i}(N) \backslash_{i+1}(N)$. Assuming $T$ countable, we may find a pure-injective $M \vDash T$ containing exactly $\aleph_{0}$ copies of $N$ when decomposed. Set $M=N_{1} \oplus \overline{N^{\left(\aleph_{0}\right)}}$. By a standard argument we may find some $a \in \overline{N^{\left(\aleph_{0}\right)}}$ such that if $b_{n}=\left(a_{0}, a_{1}, \ldots, a_{n-1}, \overline{0}\right) \in N^{\left(\aleph_{0}\right)}$, then $\varphi_{n}\left(a-b_{n+1}\right)$ holds. Since $\neg \varphi_{n}\left(b_{n+1}\right)$ holds, we see that each $a_{i}$ is dependent over 0 on $a$, but that the $a_{i}$ form an independent set. It is then not difficult to see that $N(a)-$ a direct summand of $\overline{N^{\left(\aleph_{0}\right)}}$, and so of the form $\overline{N^{(\kappa)}}$ for some $\kappa \leq \aleph_{0}$-cannot be such that $\kappa$ is finite. So $N(a) \simeq \overline{N^{\left(\aleph_{0}\right)}}$. In particular $N(a) \simeq N(a) \oplus N(a)$, so it follows easily that $M$ is not $\aleph_{0}$-homogeneous.

DEFinition 4.4. A model $M$ is $F_{\kappa}^{a}$-saturated if $M$ realizes all strong 1 -types over subsets of size $<\kappa$.

A model $M$ is weakly saturated if $M$ realizes all $n$-types over 0 , for all $n \in \omega$.

PROPOSITION 4.5. Let $M$ be superstable. Then $M$ is $F_{\aleph_{0}}^{a}$-saturated iff $M$ is pure injective and weakly saturated.

PROOF. $\Rightarrow$ Clearly if $M$ is $F_{\aleph_{0}}^{a}$-saturated then $M$ is weakly saturated.

Let $\Phi(x)$ be a set of pp formulas over $M$ : we must show that $\Phi$ is satisfied in $M$. Since $T$ is superstable, there is some $\varphi(x, \bar{a}) \in \Phi$ such that, for all $\psi(x, \bar{b}) \in \Phi$, $[\varphi(x, \overline{0}): \varphi(x, \overline{0}) \wedge \psi(x, \overline{0})]$ is finite.

Choose $c$ in $\tilde{M}$ satisfying $\Phi$. Since $M$ is $F_{\aleph_{0}}^{a}$-saturated, there is $d \in M$ with $\operatorname{stp}(c / \bar{a})=\operatorname{stp}(d / \bar{a})$. So by 3.6, we have for each $\psi(x, \bar{b}) \in \Phi$, that $\psi(c-d, \overline{0})$ holds. Therefore, from $\psi(c, \bar{b})$ and $\psi(c-d, \overline{0})$ we obtain $\psi(d, \bar{b})$, and this for each formula in $\Phi$. So $d$ satisfies $\Phi$.

$\Leftarrow$ Suppose, conversely, that $M$ is weakly saturated and pure injective. Since, by $4.3, M$ is therefore $\aleph_{0}$-homogeneous, $M$ is $\aleph_{0}$-saturated.

Let $\bar{a}$ be a finite subset of $M$ and let $c \in \tilde{M}$. We must find $d \in M$ such that $\operatorname{stp}(d / \bar{a})=\operatorname{stp}(c / \bar{a})$. Let $q=\operatorname{tp}(c / M)$. Since $M$ is pure injective, there is $d^{\prime} \in M$ satisfying $q^{+}$. Let $p=\operatorname{tp}(c / \bar{a})$.

Let $r(x)=p(x) \cup\left\{\psi\left(x-d^{\prime}\right): \psi\right.$ is pp over 0 and $\psi(x-m) \in q^{+}$for some $\left.m \in M\right\}$. Then $r$ is consistent (being realized by $c$ ), and is over $\bar{a}^{-} d^{\prime}$. Therefore, since $M$ is $\aleph_{0}$-saturated, $r(x)$ is realized in $M$, by $d$ say.

Note that $\psi(x) \in \mathcal{G}_{0}(p)$ implies that there is $m \in M$ with $\psi(c-m)$. By choice of $r$ and $d$, we have $\psi(c-d)$. Then by 3.6 it follows that $\operatorname{stp}(c / \bar{a})=\operatorname{stp}(d / \bar{a})$, as required.

Is superstability necessary in 4.5 ?

COROLlaRY 4.6. Let $M$ be superstable and $F_{\aleph_{0}}^{a}$-saturated. Then any elementary extension of $M$ also is $F_{\aleph_{0}}^{a}$-saturated.

Proof. By 4.5, $M$ is weakly saturated and pure injective. If $N \succ M$ then certainly $N$ is weakly saturated, and by 4.2 is also pure injective. So by $4.6, N$ is $F_{\aleph_{0}}^{a}$-saturated. 
5. Ziegler's theorem on superstable modules. In $[\mathbf{Z g}]$ the possible uncountable spectra for theories of modules were described. Here we give a proof of Ziegler's result using our methods.

DEFINITION 5.1. (i) Let $M \prec_{1}^{+} N$. Say that $M$ is relatively pure injective in $N$ if every set of pp formulas in $x$ over $M$ which is realized in $N$, is realized in $M$.

(ii) Let $M \prec N$. Say that $M$ is relatively $F_{\aleph_{0}}^{a}$-saturated in $N$ if, for every $\bar{a}$ in $M$ and $b \in N, \operatorname{stp}(b / \bar{a})$ is realized in $M$. (This is $M \subset_{h} N$ in Shelah [Sh2].)

As in 4.5 one may easily prove the following.

PROPOSITION 5.2. Let $T$ be superstable and let $M \prec N$. If $M$ is relatively $F_{\aleph_{0}}^{a}$-saturated in $N$, then $M$ is relatively pure injective in $N$.

A standard type of proof (one directly splits the inclusion) shows the following.

Proposition 5.3. Let $M \prec_{1}^{+} N$ with $M$ relatively pure injective in $N$. Then $N=M \oplus K$ for some $K$.

Proposition 5.4. Let $T$ be superstable, $|T|=\aleph_{0}$. Let $N \vDash T$. Then there is $M \prec N$ such that:

(i) $|M| \leq 2^{\aleph_{0}}$; and

(ii) $N=M \oplus K$, where $K$ is totally transcendental.

PROOF. First we obtain $M \prec N$ such that $|M| \leq 2^{\aleph_{0}}$ and $M$ is relatively $F_{\aleph_{0}}^{a}{ }^{-}$ saturated in $N$. This is clearly possible, since $\left|S_{n}(0)\right| \leq 2^{\aleph_{0}}$, and for any $\bar{a}$ in $M$, there can be at most $2^{\aleph_{0}}$ elements in $N$ with different strong types over $\bar{a}$.

Having obtained such an $M$, note that by $5.2, M$ is relatively pure injective in $N$, and so, by $5.3, N=M \oplus K$. By considering the invariants, $K$ is clearly totally transcendental.

Note that $K$ in 5.4 above has an essentially unique expression as a direct sum of indecomposable modules, each of which is itself totally transcendental and unlimited.

Now let $T$ be superstable and countable. Let $\mathbf{J}$ be the set of isomorphism classes of unlimited indecomposables in $\mathcal{P}(T): \mathbf{J}=\{K: K$ is indecomposable and $M \oplus K \vDash$ $T$ for some $M \vDash T\}$.

Note that if $K_{i} \in \mathbf{J}(i \in I)$, then $\bigoplus_{I} K_{i}^{\left(\lambda_{i}\right)}$ is totally transcendental and in $P(T)$ for any $\lambda_{i}$. Also, whenever $M \vDash T$ and $M \oplus K \vDash T$, then there is an essentially unique decomposition $K=\bigoplus_{I} K_{i}^{\left(\lambda_{i}\right)}$ for some $K_{i} \in \mathbf{J}$, and some $\lambda_{i}$. Set $\kappa=|\mathbf{J}|$, so (since $\bigoplus_{K \in \mathbf{J}} K$ is t.t.) $1 \leq \kappa \leq \aleph_{0}$.

THEOREM $5.5[\mathbf{Z g}]$. Let $T$ be superstable, not $\omega$-stable, with $|T|=\aleph_{0}$. Let $\lambda=\aleph_{\alpha}$. Then:

(i) if $\aleph_{0}<\lambda \leq 2^{\aleph_{0}}, I(\lambda, T)=2^{\lambda}$;

(ii) for $\lambda>2^{\aleph_{0}}, I(\lambda, T)=2^{2^{\aleph_{0}}}$, or $2^{2^{\aleph_{0}}}+|\alpha|$, or $2^{2^{\aleph_{0}}}+|\alpha|^{\aleph_{0}}$, depending on whether $\kappa$ is 1 , finite and $\neq 1$, or $\aleph_{0}$.

Proof. (i) This is Shelah [Sh1, Chapter VIII].

(ii) By 5.4 and the above remarks, any $N \vDash T$ is of the form $M \oplus K$, where $|M| \leq 2^{\aleph_{0}}, M \vDash T$ and $K=\bigoplus_{K_{i} \in \mathbf{J}} K_{i}^{\left(\lambda_{i}\right)}$, where the $\lambda_{i}$ are determined uniquely by $K$. Clearly there are at most $2^{2^{\aleph_{0}}}$ possibilities for $M$; on the other hand by [Sh1] again, for $\lambda \geq 2^{\aleph_{0}}$, one has $I(\lambda, T) \geq 2^{2^{\aleph_{0}}}$. Then if $\kappa=1,1<\kappa<\aleph_{0}, \kappa=\aleph_{0}$, 
there are respectively 1 (note $\lambda>2^{\aleph_{0}}$ ) $|\alpha|$ (if $\alpha$ is infinite), $|\alpha|^{\aleph_{0}}$ possibilities for $K$. Thus the result follows.

We leave the following as an exercise (which partly generalizes the existence of hulls).

Proposition 5.6. Let $T$ be superstable, let $M \prec N$ with $M$ relatively $F_{\aleph_{0}}^{a}$ saturated in $N$ (respectively, relatively pure injective in $N$ ). Let $A \subseteq N$. Then there is $N^{\prime} \prec N$ with $M \cup A \subseteq N^{\prime}$ such that $N^{\prime}$ is prime over $M \cup A$ among all $N^{\prime \prime} \succ M$ with $A \subseteq N^{\prime \prime}$ such that $M$ is relatively $F_{\aleph_{0}}^{a}$-saturated (resp. relatively pure injective) in $N^{\prime \prime}$. Moreover, $N^{\prime}$ is unique up to isomorphism over $M \cup A$.

6. Regular types. In this section we will be concerned with regular types: with conditions under which we may find realizations of such types, and with a method of constructing regular types (with specified properties).

Recall that a type $p \in S_{1}(A)$ is regular if $p$ is orthogonal to all its forking extensions. Therefore if $M \supseteq A$ is any model and if $q \in S_{1}(M)$ is any nonforking extension of $p \in S_{1}(A)$, then $p$ is regular iff $q$ is regular.

The following essentially appear then in $[\mathbf{P t 2}$ and $\mathbf{Z g}]$.

PROPOSITION 6.1. $p \in S_{1}(A)$ is regular iff whenever $a, b$ are nonzero elements of $N\left(p_{*}\right)$ satisfying $\left(p_{*}\right)^{+}$, then $a$ and $b$ have the same type (over 0$)$ in $N\left(p_{*}\right)$.

Note that it follows that regularity of $p \in S_{1}(A)$ depends only on $G_{0}(p)$.

COROLlaRY 6.2. If $p \in S(A)$ is regular then $N\left(p_{*}\right)$ is indecomposable: that is, $p_{*}$ is irreducible.

.ProOF. Suppose $a \in N(a)=N\left(p_{*}\right)$ realizes $p_{*}$. Let $a=\left(a_{1}, a_{2}\right) \in N_{1} \oplus N_{2}=$ $N(a)$. By 6.1, $a_{1}, a_{2}$ each has type $p_{*}$ or is 0 , and $a_{1}$ (say) is nonzero. Since $a_{1}(\neq 0) \in N(a)$, there is $(\S 0)$ a pp formula $\varphi$ with $\varphi\left(a, a_{1}\right) \wedge \neg \varphi(a, 0)$. Projecting to $N_{2}, \varphi\left(a, a_{1}\right)$ yields $\varphi\left(a_{2}, 0\right)$. Since $\neg \varphi(a, 0)$ holds, $\operatorname{tp}(a) \neq \operatorname{tp}\left(a_{2}\right)$. Hence $a_{2}=0$, as desired (since then $N_{2}=N\left(a_{2}\right)=0$ ).

It is not in general true that if $p \in S_{1}(0)$ is regular then $N(p)$ is indecomposable. For example, let $T=\operatorname{Th}\left(\mathbf{Z}_{2} \oplus \mathbf{Z}_{3}^{\left(\aleph_{0}\right)}\right)$ and let $p \in S_{1}(0)$ be the type of an element of order 6. So $N(p)=\mathbf{Z}_{2} \oplus \mathbf{Z}_{3}$, but $p$ is easily seen to be regular with $N\left(p_{*}\right)=\mathbf{Z}_{3}$.

Also note that if $p \in S_{1}(A), q \in S_{1}(B)$ are such that $\mathcal{G}_{0}(p)=\mathcal{G}_{0}(q)$ then $p, q$ are nonorthogonal; in fact, $p, q$ are RK-equivalent in the strong sense that if $p^{\prime}, q^{\prime}$ are nonforking extensions of $p, q$ to a pure-injective model $M \supseteq A \cup B$, and if $N \succ M$ is any elementary extension of $M$, then $N$ realizes $p^{\prime}$ iff $N$ realizes $q^{\prime}$ (iff $N$ realizes $\left.p_{*}=q_{*}\right)($ compare $\S 3)$.

We now consider existence of realizations of regular types. The following is due to G. Srour.

Proposition 6.3. Let $T$ be superstable, and let $M \prec N, M \neq N$ be any models of $T$. Then there is $c \in N \backslash M$ such that $\operatorname{tp}(c / M)$ is regular.

Proof. Choose $c \in N \backslash M$ such that $U(\operatorname{tp}(c / M)$ is least possible. Set $p=$ $\operatorname{tp}(c / M)$. by $2.11,2.13$ there is a pp formula $\varphi(x, \bar{m}) \in p$ with $\mathrm{D}(\varphi(x, \bar{m}))=$ $\mathrm{D}(p)=U(p)=\alpha$, say.

Consider $N(M \frown c)=\bar{M} \oplus N\left(c_{1}\right)$ say, where $c=c_{0}+c_{1}$ with $c_{0} \in \bar{M}$. Note that $\operatorname{tp}\left(c_{1} / 0\right)=p_{*}-$ so, by 6.1 , in order to show that $p$ is regular, we must show that if $d_{1} \in N\left(c_{1}\right), d_{1} \neq 0$, with $p_{*}^{+}\left(d_{1}\right)$, then $\psi\left(d_{1}\right) \Rightarrow \psi\left(c_{1}\right)$ for all pp $\psi$. 
Suppose this not so. Then given $d_{1}, \psi$ of this sort, note that $[\varphi(x, \overline{0}): \varphi(x, \overline{0}) \wedge$ $\psi(x, \overline{0})]$ is infinite (since $N\left(p_{*}\right)$ is unlimited), so $\psi(x) \notin \mathcal{G}_{0}(p)$.

Note that $d_{1} \in N(M \frown c)$, and $d_{1}$ and $M$ are direct-sum independent. Thus $d_{1}$ and $c$ are not independent over $M$ : for $d_{1} \in N\left(M \sim^{-} c\right)$ implies $\chi\left(d_{1}, c, \bar{m}\right) \wedge$ $\neg \chi\left(d_{1}, 0, \overline{0}\right)$ for some pp $\chi$ and $\bar{m}$ in $M$, and so $\chi\left(d_{1}, c, \bar{m}\right) \wedge \neg \chi\left(d_{1}, m_{1}, \bar{m}\right)$ for all $m_{1} \in M$ (since $d_{1}, M$ are direct-sum independent).

By 0.9 let $\theta$ be pp such that $\theta\left(c, d_{1}, \bar{m}\right)$ holds for some $m$ in $M$, but $\neg \theta\left(c, m_{1}, \bar{m}^{\prime}\right)$ holds for all $m_{1}, \bar{m}^{\prime}$ in $M$.

Then we have $\theta\left(c, d_{1}, \bar{m}\right) \wedge \psi\left(d_{1}\right)$. Therefore, there is $d^{\prime} \in N$ with $\theta\left(c, d^{\prime}, \bar{m}\right) \wedge$ $\psi\left(d^{\prime}\right)$. By the paragraph above it follows that $d^{\prime} \notin M$. But, since $\psi\left(d^{\prime}\right)$ holds and $[\varphi(x, \overline{0}): \psi(x)]$ is infinite, it follows from $\S 2$ that $U\left(\operatorname{tp}\left(d^{\prime} / M\right)\right)<\alpha$. This contradicts the choice of $c$.

DEFINITION 6.4. A type $p$ is finitely generated if there is $\varphi \in p^{+}$such that $p^{+} \leftrightarrow \varphi$.

PROPOSITION 6.5. If $p \in S(0)$ is isolated, then $p$ is finitely generated.

Proof. Suppose that $\chi(\bar{x})$ isolates $p$. Then we may replace $\chi$ by $\varphi(\bar{x}) \wedge$ $\bigwedge_{1}^{n} \neg \psi_{i}(\bar{x})$, where $\varphi, \psi_{1}, \ldots, \psi_{n}$ are pp.

If $p$ were not finitely generated, we could find $\varphi=\varphi_{0}>\varphi_{1}>\cdots$, all in $p^{+}$: for otherwise, for some $m \in \omega$, and for all $\varphi^{\prime} \in p^{+}$, we would have $\varphi^{\prime} \wedge \varphi_{m}=\varphi_{m}$, so would have $\varphi_{m} \leftrightarrow p^{+}$.

Now we have $\varphi \wedge \bigwedge_{1}^{n} \neg \psi_{i} \rightarrow \varphi_{m}$ for all $m$. Hence $\varphi \rightarrow \varphi_{m} \vee \bigvee_{1}^{n} \psi_{i}$. Since the indices $\left[\varphi: \varphi_{m}\right]$ are strictly increasing, from 0.2 we conclude $\varphi \rightarrow \bigvee_{1}^{n} \psi_{i}$. But then $\varphi \wedge \bigwedge_{1}^{n} \neg \psi_{i}$ would be contradictory - which is impossible - as required.

Next, we consider a general method of constructing irreducible types. We then use this method in particular cases, and obtain types which are actually regular.

PROPOSITION 6.6. Let $\Psi$ be a collection of pp formulas (over 0 ) such that $\Psi$ is a nontrivial ideal in the lattice of $p p$ formulas (subgroups), and such that if $[\varphi: \psi]$ is finite and $\psi \in \Psi$ then $\varphi \in \Psi$. Let $\Phi$ be a filter of $p p$ formulas maximal with respect to $\Phi \cap \Psi=\varnothing$.

Then there is $p \in S(0)$ with $p^{+}=\Phi$, and $p$ is unlimited and irreducible.

ProOF. Clearly there is $p \in S(0)$ with $p^{+}=\Phi,(p=\Phi \cup\{\neg \psi: \psi \in \Psi\})$. Any such type $p$ is uniimited since, if $\varphi \in \Phi$ and $\theta \notin \Phi$ were such that $[\varphi: \theta]$ finite, then since $p^{+}$is maximal, there would be $\varphi_{1} \in \Phi$ such that $\theta \wedge \varphi_{1} \in \Psi$. But then we would have $\varphi \wedge \theta \wedge \varphi_{1} \in \Psi$. But $\left[\varphi \wedge \varphi_{1}: \varphi \wedge \varphi_{1} \wedge \theta\right]$ is finite, so we would have $\varphi \wedge \varphi_{1} \in \Psi$-contradiction.

$p$ is irreducible since if $\theta_{1}, \theta_{2} \notin \Phi$ then there are $\varphi_{1}, \varphi_{2} \in \Phi$ such that $\theta_{i} \wedge \varphi_{i} \in \Psi$ $(i=1,2)$. Replace each $\varphi_{i}$ by $\varphi=\varphi_{1} \wedge \varphi_{2}$. Then we have $\varphi \wedge \theta_{1}+\varphi \wedge \theta_{2} \in \Psi$ since $\Psi$, being an ideal, is closed under " + ". Hence $p$ is irreducible by 0.15 .

We now give two applications of this result. Another application will appear in $\S 7$.

Proposition 6.7. Suppose $\operatorname{MR}(T) \geq \aleph_{0}($ possibly $\infty)$. Then there is $p \in S_{1}(0)$ which is nonisolated and irreducible. If $T$ is totally transcendental, then $p$ may further be taken to be regular.

Proof. Let $\Psi=\{\varphi: \varphi(x)$ is pp and the interval $[\varphi, 0]$ has finite length $\}$. Note (by modularity of the lattice of pp-definable subgroups) that $\Psi$ is indeed an ideal. 
By assumption and $\S 2$, " $x=x " \notin \Psi$, so we may choose $p \in S_{1}(0)$ such that $p^{+}$ is maximal not intersecting $\Psi$. By $6.6 p$ is irreducible. Moreover, $p$ is nonisolated, for if $\varphi \wedge \bigwedge_{1}^{n} \neg \psi_{i}$ isolated $p$, where we may assume $\varphi \leftrightarrow p^{+}$by 6.5 , we would have, for each $i \leq n$, that $\varphi \wedge \psi_{i} \in \Psi$.

Then $\bigvee_{1}^{n} \varphi \wedge \psi_{i} \leq \sum_{1}^{n} \varphi \wedge \psi_{i} \in \Psi$. So $\left[\sum_{1}^{n} \varphi \wedge \psi_{i}, 0\right]$ has finite length. But then, since $[\varphi, 0]$ has infinite length, there is $\theta$ pp with $\varphi>\theta>\sum_{1}^{n} \varphi \wedge \psi_{i}$. But this shows that $\varphi \wedge \bigwedge_{1}^{n} \neg \psi_{i}$ is not complete-contradiction.

If $T$ is totally transcendental, then consider $N(p)=N\left(p_{*}\right)$ - a t.t. module. Choose $q$ with $N(q) \simeq N(p)$ and such that $q^{+}$defines a minimal nonzero ppdefinable subgroup of $N(p)$. By $6.1, q$ is regular. Further, $p$ nonisolated implies that $p$ is not realized in the prime model $M_{0}$ of $T$. Since $M_{0}$ is an essentially unique direct sum of indecomposable submodules, this means that $N(p)=N(q)$ is not a direct summand of $M_{0}$ : in particular, $q$ is not realized in $M_{0}$. So $q$ is nonisolated and regular, as required.

Note that in 6.7 we need an assumption such as $\operatorname{MR}(T) \geq \aleph_{0}$ : non- $\aleph_{0}$-categoricity is not enough to ensure a nonisolated irreducible type. For example, consider $\operatorname{Th}(\mathbf{Q})$.

EXAMPLE. Let $K$ be an infinite field. Define $R$ to be the commutative ring $K\left[x_{i}(i \in \omega): x_{i} x_{j}=0(i, j \in \omega)\right]$. It is not very difficult to check that $R_{R}$ is totally transcendental, with Morley rank $\omega+1$. The type $p(v)$ obtained form 6.7 (there is only one) is one which places $v$ in the Jacobson radical $J$ of $R\left(=\sum\left\{x_{i} R: i \in \omega\right\}\right)$, so it contains $v \cdot x_{i}=0$ for each $i$, but not in any finitely generated ideal inside the radical. The hull of this type may be shown to be the simple module $R / J$.

Proposition 6.8. Let $T$ be non-totally-transcendental. Then there is $p \in$ $S_{1}(0)$ which is unlimited, not finitely generated (hence nonisolated) and regular.

ProOF. Let $\Psi=\{\varphi(x): \varphi$ is pp and the interal $[\varphi, 0]$ has the dcc $\}$. Note that $\Psi$ is an ideal and, since $T$ is not totally transcendental, $x=x \notin \Psi$. So choose an uinlimited, irreducible $p \in S_{1}(0)$ as in 6.6.

Note that $p$ is not finitely generated: for if we had some pp formula $\varphi$ with $\varphi \leftrightarrow p^{+}$, then, clearly, if $\psi<\varphi$, we would have (by maximality of $p^{+}$) $\psi \in \psi$; hence $[\psi, 0]$ would have the dcc. But then $[\varphi, 0]$ would have dcc-contradiction.

Now suppose $q \in S_{1}(A)$ is a forking extension of $p$. So, as noted above, there is $\psi \in \mathcal{G}(q) \backslash \mathcal{G}_{0}(p)$ with $\psi \in \Psi$. Let $p_{1} \in S(A)$ be a nonforking extension of $p$. Let $a$ realize $p_{1}, b$ realize $q$. To show that $p$ is regular, we must show that $a$ and $b$ are independent over $A$.

If $a, b$ were dependent over $A$, then, as above, there would be some pp formula $\theta(x, b, \bar{a})$ with $\bar{a}$ in $A$, such that $\theta(x, 0, \overline{0})$ has the dcc and $\theta(a, b, \bar{a})$ holds. Also, by the above, there is some $\psi\left(y, \bar{a}^{\prime}\right)$ pp with $\bar{a}^{\prime}$ in $A$, such that $\psi(y, \overline{0}) \in \Psi$ and $\psi\left(b, \bar{a}^{\prime}\right)$ holds. Thus the formula $\exists y\left(\theta(x, y, \bar{a}) \wedge \psi\left(y, \bar{a}^{\prime}\right)\right) \in p_{i}(x)$, whence $\chi(x)=$ $\exists y(\theta(x, y, \overline{0}) \wedge \psi(y, \overline{0})) \in p^{+}$(since $p$ is unlimited). But by $2.21, \chi$ has the dcccontradiction, as required. Hence $p$ is indeed regular.

Note that the type $p$ produced in 6.8 has $\operatorname{MR}(p)=\infty$ but $\operatorname{UR}(p)<\infty$ since every forking extension of $p$ has MR (hence UR) $<\infty$.

Examples to consider, illustrating 6.8 are: $R=\mathbf{Z}, T=\operatorname{Th}\left(\mathbf{Z}_{(p)}\right) ; R$ any left noetherian (or just left coherent) ring which is not left artinian, $T=\operatorname{Th}\left(R_{R}\right)$.

Proposition 6.9. Let $p \in S_{1}(0)$. 
(i) Let $a_{0}, a_{1}, \ldots, a_{n}$ be an independent set of realizations of $p$ all with the same strong type.

Then $\left\{a_{1}-a_{0}, \ldots, a_{n}-a_{0}\right\}$ is an independent set of realizations of $p_{*}$.

(ii) Let $b$ realize $p$, and let $\left\{a_{1}, \ldots, a_{n}\right\}$ be an independent set of realizations of $p_{*}$, such that $b$ and $\left\{a_{1}, \ldots, a_{n}\right\}$ are independent over 0 .

Then $\left\{b, a_{1}+b, \ldots, a_{n}+b\right\}$ is an independent set of realizations of $p$ and, moreover, $\operatorname{stp}\left(a_{i}+b\right)=\operatorname{stp}(b)$ for each $i$.

ProOF. (i) By 3.8, we have $\varphi\left(a_{i}-a_{0}\right)$ for each $i$ and each $\varphi \in\left(p_{*}\right)^{+}$. On the other hand, if $\varphi \notin\left(p_{*}\right)^{+}, \varphi \mathrm{pp}$, then we have $\neg \varphi\left(a_{i}-a_{0}\right)$ since $a_{i}, a_{0}$ are independent over 0 , and $\varphi \notin \mathcal{G}_{0}\left(\operatorname{tp}\left(a_{i}\right)\right)$. Therefore the $a_{i}-a_{0}$ do realize $p_{*}$.

That they are independent follows from general considerations: suppose, inductively that we have $\left\{a_{1}-a_{0}, \ldots, a_{k}-a_{0}\right\}$ an independent set. Choose a model $M$ containing $a_{0}, \ldots, a_{k}$ and independent over 0 from $a_{k+1}$. Then since $a_{0} \in M$, clearly $a_{k+1}-a_{0}$ is independent from $M$ over 0 .

(ii) That the $a_{i}+b$ realize $p$, and all have the same strong type as $b$, follows from $3.8,3.9$. Independence follows as in (i).

COROllary 6.10. Let $p \in S_{1}(0)$ be regular. Let $M$ be a model of $T$, and let $a \in M$ realize $p$. Suppose $\operatorname{dim}\left(p_{*}, M\right)=n$. Then $\operatorname{dim}(\operatorname{stp}(a), M)=n$ or $n+1$.

Proof. Note that, since $p$ is regular, so is $p_{*}$. Thus $\operatorname{dim}\left(p_{*}, M\right)$ is well defined. By 6.9(i) we have $\operatorname{dim}(\operatorname{stp}(a), M) \leq n+1$.

To show that $\operatorname{dim}(\operatorname{stp}(a), M) \geq n$, take $b_{1}, \ldots, b_{n}$ to be an independent set of realizations of $p_{*}$ in $M$. If we let $B$ denote a maximal subset of $\left\{b_{1}, \ldots, b_{n}\right\}$ such that $a$ and $B$ are independent over 0 , then it follows, since $p$ has weight one, that $B \supseteq\left\{b_{1}, \ldots, b_{n}\right\} \backslash\left\{b_{i}\right\}$ for some $i$. So then, by 6.9(ii), $\{a\} \cup\{a+b: b \in B\}$ is an independent set of realizations of $\operatorname{stp}(a)$ in $M$, so $\operatorname{dim}(\operatorname{stp}(a), M) \geq n$, as required.

Examples where $\operatorname{dim}\left(p_{*}, M\right)=n$ and $\operatorname{dim}(\operatorname{stp}(a), M)=n+1$ are not difficult to find: take $p=\operatorname{tp}\left(1_{\mathbf{Z}_{(p)}}\right)$ in $\operatorname{Th}\left(\mathbf{Z}_{(p)}\right)$, and $M=\mathbf{Z}_{(p)} \oplus \mathbf{Q}^{(n)}\left(\right.$ note $p_{*}=\operatorname{tp}\left(1_{\mathbf{Q}}\right)$ ). Are there examples of the other case in 6.10 ?

7. Modules with $U$-rank 1. Recall that we say that $T$ has $U$-rank $1, U(T)=$ 1 , if $T$ is not the theory of a finite structure and, for all $p \in S_{1}(0), U(p) \leq 1$. In particular, such $T$ is superstable. The next result is immediate from the characterization of $U$-rank in $\S 2$.

PROPOSITION 7.1. T has $U$-rank 1 iff for every pp formula $\varphi(x)$, either $[x=$ $x: \varphi(x)]$ or $[\varphi(x): x=0]$ is finite.

PROOF. If both were infinite then clearly $G_{0}(x=x)>G_{0}(\varphi(x))>0$, and so by 2.10, $U(T) \geq 2$ - contradiction.

Here, by $G_{0}(\varphi)$ we mean $\bigcap\left(\mathcal{G}_{0}(\varphi)=\{\psi: \psi\right.$ is pp and $[\varphi: \varphi \wedge \psi]$ is finite $\left.\}\right)$.

Examples of such theories include the theories of the following $\mathbf{Z}$-modules: $\mathbf{Z}$; $\mathbf{Z}_{(p)}^{(n)}$ for $n \geq 1 ; \mathbf{Z}_{2}^{\left(\aleph_{0}\right)} ; \mathbf{Z}_{4} \oplus \mathbf{Z}_{2}^{\left(\aleph_{0}\right)} ;$ the last two being totally transcendental. Another is the example after 6.7, but taken with $K$ a finite field. Yet another example is the "canonical example" of a superstable non-tt module with only countably many models (see $[\mathbf{Z g}, 10.3(3)]$ ). We show here how one obtains a structure theory for arbitrary models of a $U$-rank 1 countable theory of modules (although our methods 
do not actually give the Vaught conjecture here) (see added comments at the end of the paper).

Remember that one has the following situation for modules: for superstable $T$, there is a nice classification of the pure-injective modules; for $T$ totally transcendental, this becomes a classification of all the models. The problem is to have a structure theory for all the models in the superstable, non-totally-transcendental, case (observe that as far as the uncountable models are concerned, the results of $\S 5$ are sufficient for counting).

Note that for superstable $T$, the number of dimensions, $\mu(T)$, of $T$ is the number of nonorthogonality classes of regular types, and this in turn equals the number of nonorthogonality classes of unlimited regular types over 0 . This is the same as the number of nonisomorphic unlimited indecomposable (nonzero) modules in $P(T)$.

LEMMA 7.2. Let $U(T)=1$. Then there is a unique unlimited type $p \in S_{1}(0)$.

ProOF. This is immediate from 7.1: just note that

$$
\begin{aligned}
\mathcal{G}_{0}(x=x) & =\{\varphi(x): \varphi \text { is pp and }[x=x: \varphi(x)] \text { is finite }\} \\
& =\{\varphi(x): \varphi(x) \text { is nonalgebraic }\}
\end{aligned}
$$

by 7.1. Thus $p \in S_{1}(0)$ with $p^{+}=\mathcal{G}_{0}(x=x)$ is the unique unlimited type in $S_{1}(0)$.

Of course $U(p)=1$ and $p$ is regular (and $N(p)$ has no nontrivial definable subgroups). Also, notice that this is another example of the construction of 6.6.

COROllary 7.3. $U(T)=1$ implies $T$ is unidimensional.

Now, we prove a partial converse: we show that a unidimensional theory of modules is superstable (see added comments at the end of the paper). For general reasons [Ls2] it then follows that $T$ has finite $U$-rank, and examples such as $\mathbf{Z}_{2^{n}}^{\left(\aleph_{0}\right)}$ show that any finite $U$-rank (even MR) is realized in unidimensional theories of modules.

Recall $[\mathbf{Z g}]$ that $\varphi / \psi$ is a minimal pair (in $p)$ if $\left(\varphi \in p^{+}, \psi \in p^{-}\right) \varphi>\psi$ and there is no pp $\theta$ with $\varphi>\theta>\psi$. We will use a few facts from $[\mathbf{Z g}]$ about this concept.

PROPOSITION 7.4. Suppose $T$ is unidimensional (in the sense that there is an indecomposable $N \in \mathcal{P}(T)$ such that every unlimited $N_{1} \in \mathcal{P}(T)$ has the form $\overline{N^{(\kappa)}}$ for some $\kappa)$. Then $T$ is superstable.

PROOF. Let $T_{1}=\operatorname{Th}\left(N^{\left(\aleph_{0}\right)}\right)$. We show that if $T$ were not superstable, then neither would be $T_{1}$. So let $\varphi>\psi$ be pp formulas with $\operatorname{Inv}(T, \varphi, \psi)$ infinite. We show $\operatorname{Inv}\left(T_{1}, \varphi, \psi\right)$ is infinite also.

We have, working in $T$, that $G_{0}(\varphi)>G_{0}(\psi)$ since $[\varphi: \psi]$ is infinite. Hence there is an unlimited $p \in S_{1}(0)$ with $p^{+}=\mathcal{G}_{0}(\varphi)$, and, note, $\psi \notin p^{+}$. Since $p$ is realized in some unlimited $N_{1} \in P(T)$, we have $\operatorname{Inv}\left(N_{1}, \varphi, \psi\right)>1$. Since all invariants of $T_{1}$ are 1 or $\infty$, we do have $\operatorname{Inv}\left(T_{1}, \varphi, \psi\right)$ infinite.

So, it will be enough to show that $T_{1}$ is superstable, equivalently $\left(T_{1}=T_{1}^{\aleph_{0}}\right)$ is totally transcendental.

Suppose not, then $T_{1}$ contains an infinite descending chain of pp formulas. Use the construction of 6.8 to obtain an irreducible $p_{1} \in S_{1}^{T_{1}}(0)$ which, by the argument 
of 6.8, contains no minimal pair. By $[\mathbf{Z g}, 6.9$ and unidimensionality; Remark 1 on p. 192 applied to $v=v / v=0 ; 8.11 ; 4.8]$ there is some irreducible $q \in S_{1}^{T_{1}}(0)$ which does contain minimal pair. So, by $[\mathbf{Z g}, 8.10] N(q) \not N(p)$. This contradicts unidimensionality of $T_{1}$, as required.

Note that, by the proof above, finiteness of $U(T)$ is equivalent to finiteness of $\operatorname{MR}\left(T_{1}\right)$, and this is a direct consequence of 6.7 .

We now continue with the $U$-rank 1 case, obtaining a structure theorem for the models.

For the rest of this section, we make the following:

Assumption. $T$ is a countable, superstable, non- $\omega$-stable theory of modules with $U$-rank 1 (note $T \omega$-stable and of $U$-rank 1 implies $T \aleph_{1}$-categorical). Let $p$ be the unique unlimited type in $S_{1}(0)(7.2)$.

ClaIM 7.5. For every pp formula $\varphi(x)$ with $[\varphi: 0]$ infinite (so $[x=x: \varphi(x)]$ finite), there is $\psi(x)$ pp with $\varphi>\psi$ and $[\varphi: \psi]$ finite.

Proof. We know that, $T$ being non- $\omega$-stable, there is an infinite properly descending chain $\varphi_{1}(x)>\varphi_{2}(x)>\cdots$ where, note, every $\varphi_{i}$ has finite index in $x=x$. Since the indicies $\left[x=x: \varphi_{i}(x)\right]$ are strictly increasing and since $[x=x: \varphi(x)]$ is finite, eventually we have $\varphi>\varphi \wedge \varphi_{i}$ (and of course, $\left[x=x: \varphi \wedge \varphi_{i}\right]$ is finite).

CONCLUSION 7.6. $p$ is not finitely generated (so, in particular, is nonisolated); moreover, $p$ contains no minimal pair (by 7.1).

LEMMA 7.7. Let $M \oplus N(p)^{(\kappa)} \vDash T\left(M \prec_{1}^{+} \tilde{M}\right.$ arbitrary). Then $M \vDash T$ (and hence $\left.M \prec M \oplus N(p)^{(\kappa)}\right)$.

Proof. We check the invariants. So, suppose first that $\psi<\varphi$ are pp and $\operatorname{Inv}(T, \varphi, \psi)=n \in \omega$. Then, since $N(p)$ is unlimited, $\operatorname{Inv}(N(p), \varphi, \psi)=1$. But $\operatorname{Inv}(M, \varphi, \psi) \cdot \operatorname{Inv}\left(N(p)^{(\kappa)}, \varphi, \psi\right)=n$. Hence $\operatorname{Inv}(M, \varphi, \psi)=n$.

Now suppose that $\operatorname{Inv}(T, \varphi, \psi)$ is infinite. Hence $[x=x: \varphi(x)]$ is finite and $[\psi: 0]$ is finite. From the argument of 7.5 (and modularity) we can find a strictly descending chain, $\varphi=\varphi_{0}>\varphi_{1}>\cdots>\psi$. Note that each $\left[\varphi: \varphi_{i}\right]$ is finite.

From the first paragraph, we conclude that, for each $i \in \omega, \operatorname{Inv}\left(M, \varphi_{i}, \varphi_{i+1}\right)>1$. Hence $\operatorname{Inv}(M, \varphi, \psi)$ is infinite, as required.

The next result does not need $U(T)=1$, nor even superstability (see $[\mathbf{Z g}, 9.2]$, but we give a direct proof here).

COROLlaRY 7.8. Thas a prime pure-injective model $M_{0}$; that is, $M_{0} \vDash T$ is pure injective, and embeds as a direct summand in every pure-injective model of $T$.

Proof. Let $M$ be any pure-injective model of $T$. Then by 0.14 and after 0.16 , we may write $M=M_{0} \oplus N(p)^{(\kappa)}$ where $M_{0}$ contains no direct summand isomorphic to $N(p)$. By 7.7, $M_{0} \vDash T$. Note that $M_{0}$ has no unlimited direct summand: in particular, each summand of $M_{0}$ occurs a fixed, by 0.14 , finite numbr of times. So, by 1.6 (for which a direct proof here is not difficult), $M_{0}$ is an invariant of $T$, as required.

Therefore, every pure-injective model of $T$ has the form $M_{0} \oplus N(p)^{(\kappa)}$ for some $\kappa \geq 0$.

LEMMA 7.9. Let $M \vDash T$. Then $M \prec M_{0}$ iff $M$ omits $p$. 
Proof. First we establish the following:

ClaIM. Let $M \vDash T$ omit $p$. Then $\bar{M}$ omits $p$.

ProOf OF Claim. Suppose not, so $\bar{M}$ realizes $p$, say $a \in \bar{M} \backslash M$ realizes $p$. Now $U(p)=1$, so $a$ and $M$ are independent over 0 , but since $a \in \bar{M}=N(\bar{M})$, and since $p$ is unlimited, this contradicts 1.5 .

PROOF OF 7.9. $\Rightarrow$ It is enough to show that $M_{0}$ omits $p$. Since $p$ is nonisolated by 7.6 , there is $M \vDash T$ omitting $p$. But then the claim implies $\bar{M}$ omits $p$. Hence, by $7.8, M_{0}$ omits $p$, as required.

$\Leftarrow$ Let $M$ omit $p$. Then, by the claim, $\bar{M}$ omits $p$. By $7.8, \bar{M}=M_{0} \oplus N(p)^{(\kappa)}$ for some $\kappa$. Hence $\kappa=0$. So $\bar{M}=M_{0}$, and hence $M \prec M_{0}$, as required.

Proposition 7.10. Let $T$ be countable, non- $\omega$-stable, with $U(T)=1$. Then any model $M$ of $T$ may be written (essentially uniquely) as $M_{0}^{\prime} \oplus N(p)^{(\kappa)}$, where $M_{0}^{\prime} \prec M_{0}=$ the prime pure-injective model of $T$, and $p$ is the unique unlimited type in $S_{1}(0)$.

Proof. Let $\left\{a_{i}: i<\kappa\right\}$ be a maximal independent set of realizations of $p$ in $M$. Note that, if $a$ realizes $p$, if $N(a)$ is some copy of the hull of $a$, and if $b \in N(a)$, then, since $b$ and $a$ are not independent over 0 (by 1.5), $b$ is algebraic over $a$.

Therefore, $M$ contains a (in fact, every) copy $N\left(a_{i}\right)$ of the hull of each $a_{i}$. Since the $a_{i}$ are independent, so are their hulls ([Pt2] or directly) and hence we have $\bigoplus_{i} N\left(a_{i}\right)$ as a pure submodule of $M$. But since $\bigoplus_{i} N\left(a_{i}\right) \simeq N(p)^{(\kappa)}$ is totally transcendental, we have $M=M_{0}^{\prime} \oplus \bigoplus_{i} N\left(a_{i}\right)$ for some $M_{0}^{\prime}$.

By 7.9, we will be finished when we show that $M_{0}^{\prime}$ omits $p$. If this were not the case, say $a \in M_{0}^{\prime}$ realizes $P$, then by $1.5,\{a\} \cup\left\{a_{i}: i<\kappa\right\}$ would be an independent set-contradicting maximality of $\left\{a_{i}: i<\kappa\right\}$.

So the result is proved.

CONJECTURE 7.11. T a countable theory of modules, non-w-stable, with $U$ rank 1. Then $I\left(\aleph_{0}, T\right)=2^{\aleph_{0}}$ or $M_{0}$ is atomic.

Note that this would give a nice re-proof of Vaught's conjecture for superstable modules of $U$-rank 1, because in this ccase, if $M \vDash T$ is countable, and $M_{0}^{\prime} \prec M$ is as in 7.10 , then $M_{0}^{\prime}$ is just the prime model $M_{0}$ of $T$, whence $M$ is determined up to isomorphism by the number, $\kappa$, of copies of $N(p)$.

Note that there are cases (e.g. $T=\operatorname{Th}\left(\mathbf{Z}_{(2)}\right)$, and observe that $\left|S_{2}^{T}(0)\right|=2^{\aleph_{0}}$ ), where $U(T)=1$ and $I\left(\aleph_{0}, T\right)=2^{\aleph_{0}}$.

Added August 1986. 1. Buechler now has a proof of Vaught's conjecture for $U$-rank 1 modules.

2. Hrushovsky has shown that unidimensionality implies superstability for any complete theory.

\section{REFERENCES}

[Ba] W. Baur, Elimination of quantifiers for modules, Israel J. Math. 25 (1976), 64-70.

[Fi] E. Fisher, Abelian structures, Yale Univ., 1975, first part published as Abelian structures. I, in Lecture Notes in Math., vol. 616, Springer-Verlag, Berlin, 1977.

[Ga1] S. Garavaglia, Direct product decomposition of theories of modules, J. Symbolic Logic 44 (1979), $77-88$.

[Ga2] _ Decomposition of totally transcendental modules, J. Symbolic Logic 45 (1980), 155-164. 
[Ga3] _ Dimension and rank in the model theory of modules, preprint, Univ. of Michigan, East Lansing, 1980.

[Ga4] _ _ Forking in modules, Notre Dame J. Formal Logic 22 (1981), 155-162.

[Ho] W. Hodges, Modules and abelian groups (in preparation).

[Ls1] D. Lascar, Ranks and definability in superstable theories, Israel J. Math. 23 (1976), 53-87.

[Ls2] _ Relation entre le rang $U$ et le poids, Fund. Math. 21 (1984), 117-123.

[LP] D. Lascar and B. Poizat, An introduction to forking, J. Symbolic Logic 44 (1979), 330-350.

[Mc] A. Macintyre, On $\omega_{1}$-categorical theories of fields, Fund. Math. 71 (1971), 1-25.

[Mo] L. Monk, Elementary-recursive decision procedures, PhD. thesis, Univ. of California, Berkeley, 1975.

[PP] A. Pillay and M. Prest, Forking and pushouts in modules, Proc. London Math. Soc. (3) 46 (1983), 365-384.

[Po] B. Poizat, Sous-groupes définissables d'un group stable, J. Symbolic Logic 46 (1981), 137-146.

[Pt1] M. Prest, Pure-injectives and T-injective hulls of modules, Bedford College, 1981, preprint.

[Pt2] _ The generalized RK order, orthogonality and regular types for modules, J. Symbolic Logic 50 (1985), 202-219.

[R] D. J. S. Robinson, Finiteness conditions and generalized soluble groups, Vol. I, Springer-Verlag, Berlin, 1972.

[Ro1] P. Rothmaler, Total transzendente abelsche Gruppen und Morley-Ran, Report des Zentralinstituts für Math. und Mechanik, Akad. Wissenschaften der DDR, Berlin, 1978.

[Ro2] _ Some model theory of modules. II. On stability and categoricity of flat modules, J.Symbolic Logic 48 (1983), 970-985.

[Ro3] _ Stationary types in modules Z. Math. Logik Grundlag. Math. 29 (1983), 445-464.

[Sg1] G. Sabbagh, Aspects logiques de la pureté dans les modules, C. R. Acad. Sci. Paris 271 (1970), 909-912.

[Sg2] _ Sous-modules purs, existentiellement clos et élémentaires, C. R. Acad. Sci. Paris 272 (1971), 1289-1292.

[Sh1] S. Shelah, Classification theory, North-Holland, Amsterdam, 1978.

[Sh2] _ , The spectrum problem. IV, preprint 1982

[Zg] M. Ziegler, Model theory of modules, Ann. Pure Appl. Logic 26 (1984), 149-213.

[Zi] B. Zilber, The structure of models of uncountably categorical theories, preprint 1983.

[Zm] W. Zimmermann, Rein injektive direkte Summen von Moduln, Comm. Algebra 5 (1977), 10831117.

Department of Mathematics, University of Notre Dame, Notre Dame, IndiANA 46556 (Current address of Anand Pillay)

Department of Mathematics, Yale University, Box 2158, Yale Station, New HAVEN, CONNECTICUT 06520

Current address (Mike Prest): Department of Pure Mathematics, Univeristy of Liverpool L69 2BX, England 